\title{
Río Bec. Des grandes maisons et des récoltes
}

Río Bec. Big houses and staple production

Río Bec. Grandes residencias y cosechas

Marie-Charlotte Arnauld, Dominique Michelet, Chloé Andrieu, Alfonso Lacadena, Éva Lemonnier, Boris Vannière, Philippe Nondédéo et Julie Patrois

\section{OpenEdition}

\section{Journals}

Édition électronique

URL : https://journals.openedition.org/jsa/14042

DOI : 10.4000/jsa. 14042

ISSN : 1957-7842

Éditeur

Société des américanistes

Édition imprimée

Date de publication : 31 décembre 2014

Pagination : 107-144

ISSN : 0037-9174

Référence électronique

Marie-Charlotte Arnauld, Dominique Michelet, Chloé Andrieu, Alfonso Lacadena, Éva Lemonnier, Boris Vannière, Philippe Nondédéo et Julie Patrois, "Río Bec. Des grandes maisons et des récoltes »,

Journal de la Société des américanistes [En ligne], 100-2 | 2014, mis en ligne le 01 janvier 2016, consulté le 03 septembre 2022. URL : http://journals.openedition.org/jsa/14042 ; DOI : https://doi.org/10.4000/ jsa. 14042 


\title{
RÍO BEC. DES GRANDES MAISONS ET DES RÉCOLTES
}

\author{
Marie-Charlotte ARNAULD, Dominique MICHELET, Chloé ANDRIEU, \\ Alfonso LACADENA, Éva LEMONNIER, Boris VANNIÈRE, \\ Philippe NONDÉDÉO, Julie PATROIS*
}

\begin{abstract}
Dans la région de Río Bec, les données archéologiques, iconographiques et épigraphiques indiquent que la royauté n'a pas vraiment eu cours de 550 à 950 apr. J.-C. Des élites nobles, en revanche, furent d'importants acteurs, construisant des résidences à forte visibilité et contrôlant des domaines agricoles, ce qui servit leurs stratégies de factions et, tardivement, la diffusion d'idées architecturales sur une large zone. L'image que l'on reconstitue est celle d'une société fortement fragmentée entre de nombreux acteurs économiquement indépendants : chacun poursuivait ses intérêts de groupe lesquels combinaient une production vivrière intensive et l'auto-édification de résidences stables sur chaque domaine, mais, à l'inverse, peu d'investissement dans la production artisanale et dans les échanges commerciaux. [Mots-clés : Mayas, Classique récent-terminal, Basses Terres, architecture, auto-construction, agriculture infield.]
\end{abstract}

Río Bec. Big houses and staple production. In the Río Bec region, archaeological, iconographic and epigraphic data indicate that kingship has been weakly articulated from AD 550 to 950 . Sub-royal elites were active, creating highly visible houses with land domains that served factional strategies and, late in time, diffused architectural ideas over a wide area. The picture is one of a highly fragmented society consisting of many economically autonomous agents, each pursuing corporate interests that combined intensive staple production and auto-construction of stable residences on their land, with minimal insertion in craft production and regional exchanges. [Key words: Mayas, Late-Terminal Classic, Lowlands, architecture, auto-construction, infield agriculture.]

Río Bec. Grandes residencias y cosechas. En la región de Río Bec, los datos arqueológicos, iconográficos y epigráficos indican que la realeza no existió verdaderamente entre 550 y 950 d.C. En cambio, elites nobles fueron actores importantes, edificando residencias de alta visibilidad y controlando dominios agrícolas. Estas realizaciones servían sus estrategias como facciones y propiciaron a la vez, pero más tarde, la difusión de ideas arquitectónicas en una amplia zona. La imagen que se logra reconstruir es la de

* UMR 8096, Archéologie des Amériques, CNRS/université Paris 1 Panthéon-Sorbonne, 21, allée de l'université, 92023 Nanterre Cedex [charlotte.arnauld@mae.u-paris10.fr].

Journal de la Société des américanistes, 2014, 100-2, pp. 107-144. C Société des américanistes. 
una sociedad muy fragmentada entre numerosos actores económicamente independientes: cada uno perseguía sus intereses de grupo, los cuales combinaban una producción agrícola intensiva y la auto-construcción de residencias estables en su dominio. En cambio, invirtieron poco en las actividades artesanales y los intercambios comerciales. [Palabras claves: mayas, Clásico tardío-terminal, Tierras Bajas, arquitectura, auto-construcción, agricultura infield.]

\section{INTRODUCTION}

Au-delà d'un site archéologique spécifique, le terme « Río Bec » s'est appliqué à un style architectural bien individualisé, correspondant à une période, le Classique récent-terminal (600-900 apr. J.-C.), et une région, le Centre-Sud de la péninsule du Yucatán au Mexique (Figure 1). C'est géographiquement le plus enclavé des grands styles mayas classiques, avec le Chenes voisin, juste au nord. Par contraste, la tradition Petén au sud (Guatemala) s'est épanouie depuis le Préclassique (600 av. J.-C.-200 apr. J.-C.) dans la vaste région des Basses Terres du Sud ouverte sur différents milieux montagneux et fluviaux, tandis que le Puuc à l'extrême nord de la péninsule, aussi tardif que Río Bec (700-1000 apr. J.-C.), est situé plus près des milieux côtiers, favorables aux interactions socio-économiques (commerce et exploitation du sel notamment). Le style Río Bec est un phénomène localisé, à distance des puissantes capitales de Calakmul à l'ouest et Dzibanché à l'est ; il concerne une région d'une surface d'environ $6500 \mathrm{~km}^{2}$ longtemps isolée (Nondédéo et al. 2010). De fait, plusieurs des premiers groupes monumentaux découverts à l'extrême fin du XIX ${ }^{\mathrm{e}}$ siècle et au début $\mathrm{du} \mathrm{XX}^{\mathrm{e}}$ siècle dans ce qui fut considéré comme le site de Río Bec ont été ensuite perdus dans la forêt (Merwin 1913 ; Périgny 1908 ; Ruppert et Denison 1943 ; Sapper 1895a et b), et c'est seulement en 1973 que fut redécouvert le Groupe B de ce lieu (Seuffert 1974). Temporellement, le style Río Bec est limité, dans son apogée, à une période de quatre siècles, entre 550 et 950 apr. J.-C. (Taladoire et al. 2013).

L'architecture Río Bec se caractérise par de grands édifices (jusqu'à $60 \mathrm{~m}$ de long) à chambres multiples équipées de banquettes maçonnées, aux façades décorées de mosaïques de pierres, parfois flanquées de hautes tours qui imitent des temples/pyramides mais ne sont que des trompe-l'œil. Ce qui fait également l'originalité de la région Río Bec, c'est la quasi-absence de catégories d'édifices comme les vrais temples/pyramides ou les terrains de jeu de balle, si caractéristiques pourtant des grands sites mayas. Enfin, les sites Río Bec se différencient de la majorité des autres établissements mayas contemporains par leur éclatement en groupes monumentaux distincts et leur manque apparent de structuration spatiale, contribuant à la difficulté de leur localisation par les prospecteurs en dépit de leur bon état de conservation. Dans les années 1980, plusieurs archéologues mexicains réussirent à localiser à nouveau treize des groupes inventoriés au début $\mathrm{du} \mathrm{Xx}^{\mathrm{e}}$ siècle dans le site éponyme du style et de la région (Carrasco 


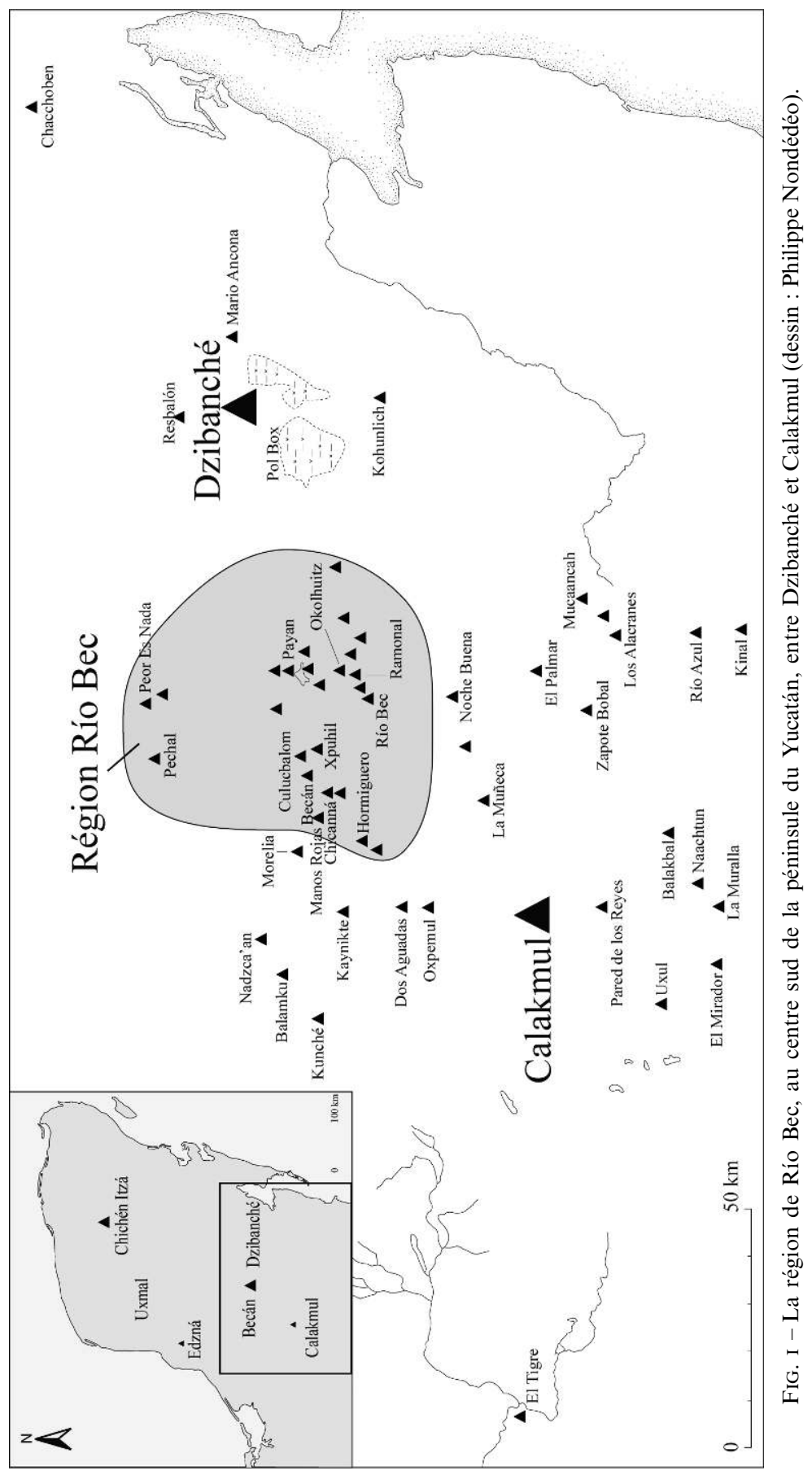


et al. 1986a et b). Associés à cette architecture spectaculaire, les vestiges assez denses d'un système agraire caractérisé par des terrasses et des levées de pierres et de terre linéaires ont été repérés en surface, au départ par l'archéologue P. M. Thomas (1981) et le géographe B. L. Turner II (1983) ; ces aménagements sont, de fait, mieux conservés, plus étendus qu'ailleurs dans les Basses Terres et on les observe à l'intérieur même des établissements. Sur ces bases, le site de Río Bec proprement dit a fait l'objet d'interprétations socio-économiques et politiques (Adams 1981 ; Adams et Jones 1981) alors même qu'il était mal reconnu et que son originalité n'était pas bien évaluée.

La région Río Bec constituait encore récemment un des secteurs les plus énigmatiques de l'aire maya. Les singularités que l'on vient d'évoquer demandaient à être vérifiées et interprétées dans le site éponyme de Río Bec, le plus vaste ensemble de groupes monumentaux de la région à la fin du Classique. Développé entre 2002 et 2010, le projet Río Bec visait à élucider les conditions environnementales, économiques, sociales et politiques de l'apogée de ce site. Pour ce faire, et entre autres, cinq grandes opérations ont été conduites (Michelet et al. 2004b, 2005, 2006, 2007, 2008 ; Nondédéo et al. 2002 ; voir aussi Fowler et Arnauld 2013) :

- une reconnaissance micro-régionale par informateurs et parcours prédéterminés sur carte à l'échelle de $100 \mathrm{~km}^{2}$ autour de l'ensemble le plus dense de groupes monumentaux connus en 2002 (ensemble qui comprend les groupes codés A, B, C, D, E, G, H, J, K, L M) (Figure 2) (Nondédéo et al. 2013) ;

- une prospection systématique à l'échelle des 159 ha que couvre cet ensemble (Figure 2) (Nondédéo et al. 2013) ;

- des opérations de fouilles intensives et extensives qui ont dégagé intégralement 23 bâtiments appartenant aux trois groupes monumentaux A, B et D et à six unités domestiques voisines (Figure 3) (Arnauld et al. 2013, Michelet et al. 2013) ;

- deux programmes de sondages (169 au total) à des fins chronologiques aux deux échelles mentionnées, $100 \mathrm{~km}^{2}$ et 159 ha (la proportion totale des unités datées de la sorte, qu'elles aient été monumentales ou non, avoisinant les $40 \%$ : Taladoire et al. 2013) ;

- enfin, un relevé micro-topographique précis d'un secteur-test de 8 ha autour de l'un des groupes fouillés, accompagné de fouilles exploratoires de toutes les catégories de structures présentes, tant agricoles que résidentielles (Figure 5) (Lemonnier et Vannière 2013).

Pour explorer les relations qui ont existé entre les groupes monumentaux, les unités domestiques et les vestiges du parcellaire agricole dans le contexte de l'environnement local, les résultats des différentes opérations de terrain réalisées seront ici brièvement synthétisés en ce qui concerne l'architecture, les structures de l'habitat à l'échelle de la micro-région $\left(100 \mathrm{~km}^{2}\right)$ et de la zone nucléaire (159 ha) ainsi que la séquence chrono-céramique d'occupation. Cela, afin de 


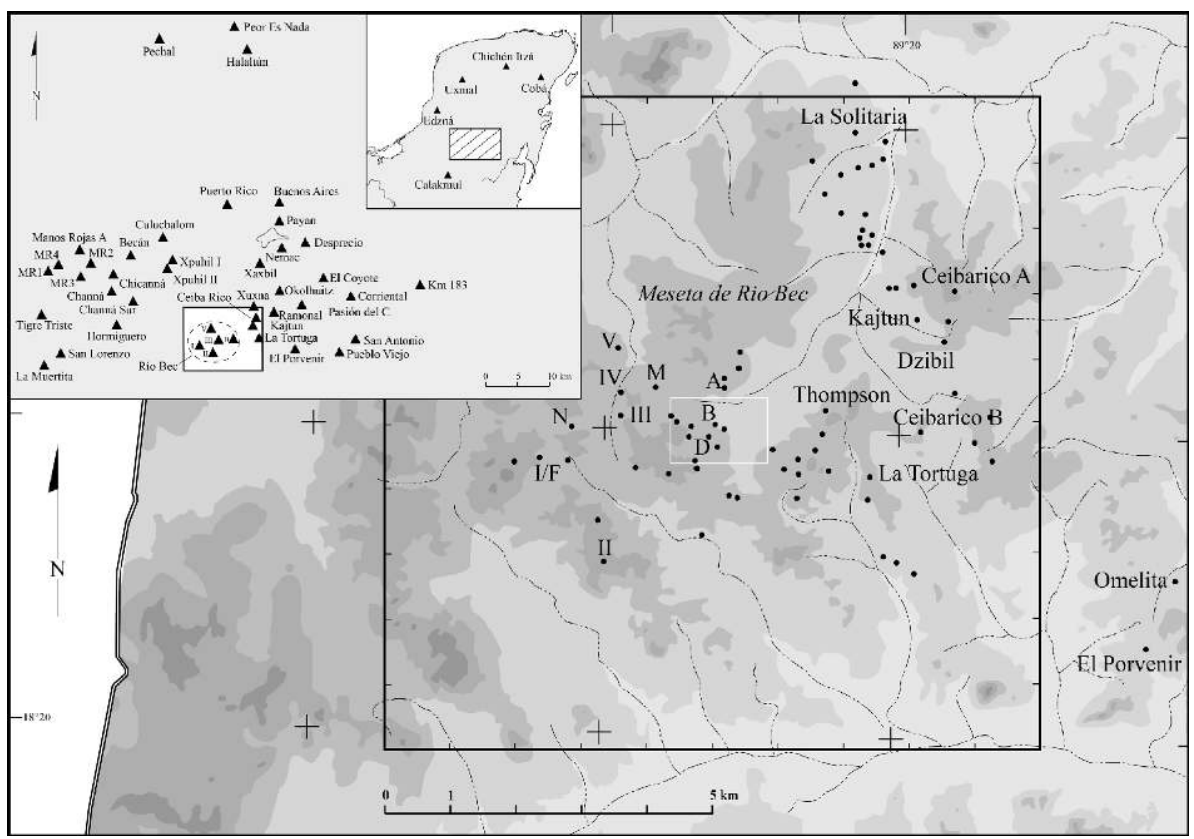

FIG. 2 - Centre de la micro-région d'étude de $100 \mathrm{~km}^{2}$; au centre de celle-ci, la zone nucléaire de 159 ha, systématiquement et intégralement prospectée (dessin : Philippe Nondédéo).

traiter ensuite la question de l'économie domestique. En d'autres termes, partant de ce qui était (mal) connu de Río Bec au début du projet - un style architectural -, il s'agit de montrer quelles sont les unités sociales et politiques qui étaient en jeu localement dans cette région politiquement et économiquement isolée ; quel fut leur développement dans le temps et comment comprendre leur fonctionnement au niveau de l'unité domestique élémentaire de la société, celle où s'organisait l'exploitation de l'unité de production agricole et les échanges supposés. Compte tenu de la faiblesse des gouvernants, des institutions politiques communautaires et des structures de l'habitat qui auraient résulté de leurs effets, l'étude des relations économiques dans le secteur de Río Bec permet d'écarter l'idée d'un « contrôle économique par les élites » (voir, par exemple, Wells 2006, pp. 272-273). Pour autant, la société Río Bec n'était pas égalitaire et ses groupes constitutifs, autonomes, avaient des modes de production et d'échange plus complexes qu'il n'y paraît. Ces modes ont été fortement influencés par les contraintes de l'architecture monumentale résidentielle, sa production et son entretien : à ce niveau, notre étude suggère que les effets socio-techniques de cette architecture dans les sociétés mayas du Classique récent-terminal ont été sousestimés jusqu'à présent. 


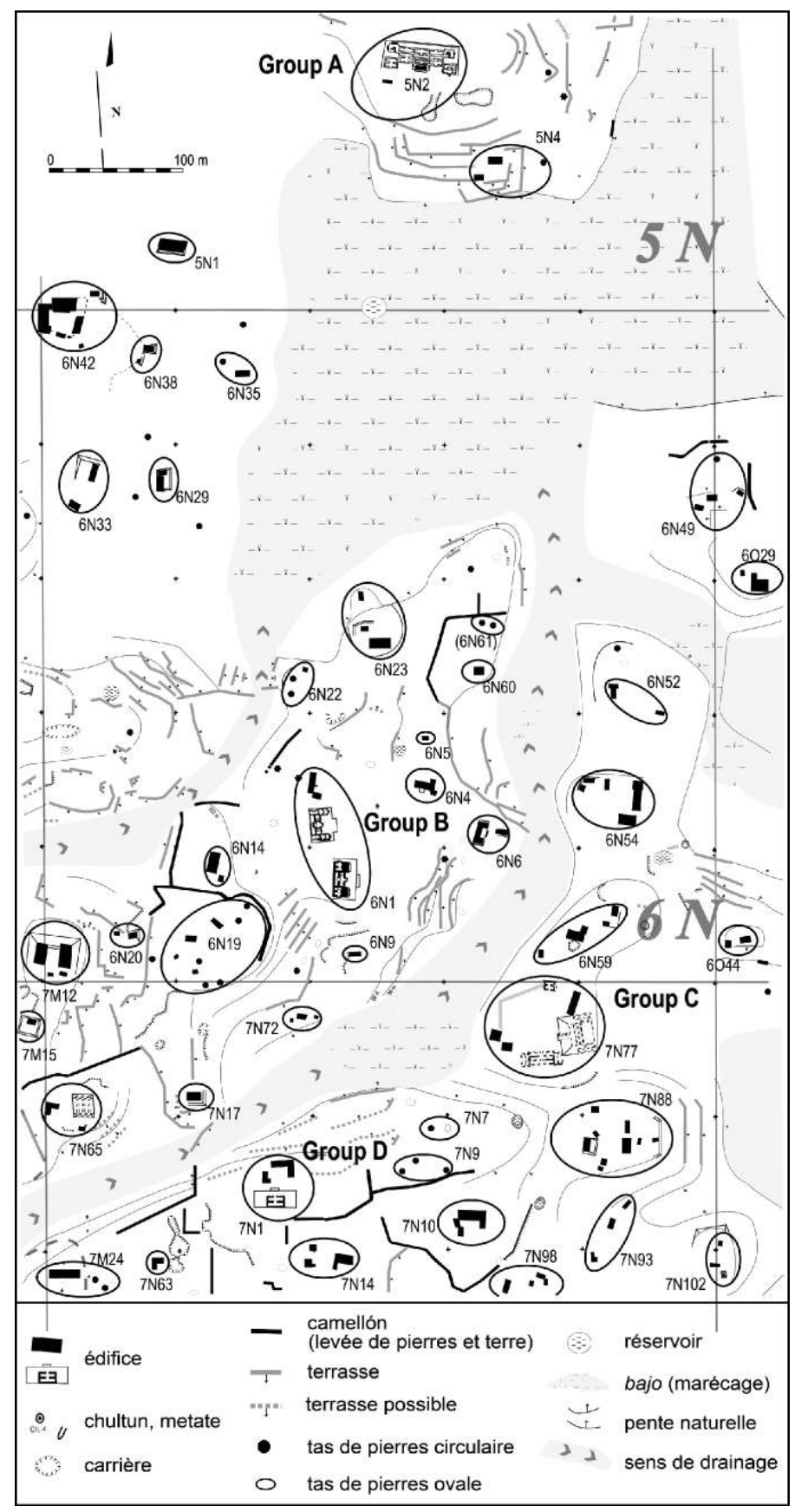

FIG. 3 - Partie centrale de la zone nucléaire de Río Bec avec délimitation des unités résidentielles par des cercles et des ellipses (dessin : Philippe Nondédéo, Dominique Michelet, Éva Lemonnier). 


\section{UN STYLE ARCHITECTURAL RÉSIDENTIEL : LES GRANDES MAISONS Río BEC}

Une bonne vingtaine de groupes monumentaux (25 excatement) étaient connus à l'échelle de la micro-région au début du projet Río Bec (2002), dont plusieurs étaient à relocaliser. Avec les deux prospections réalisées en 2002 et 2003, 48 nouveaux groupes de ce type ont été découverts (total : 73). De plus, dans la zone nucléaire de 159 ha, une centaine d'unités d'habitat plus modestes ont été identifiées et relevées (Michelet et al. 2004b ; Nondédéo et al. 2002). Les groupes monumentaux sont définis par la présence d'au moins un grand édifice multi-pièces parmi les monticules spatialement associés qui les composent. La distance moyenne qui sépare deux groupes monumentaux dans la partie des $100 \mathrm{~km}^{2}$ reconnue est de $350 \mathrm{~m}$. Parmi les 73 groupes recensés, un seul, Kajtún, comporte 62 « structures » (bâtiments ou monticules), un autre, le Groupe I, en comporte 34, et le Groupe V, 18, les autres ayant chacun moins de 10 structures groupées. Les unités de rangs inférieurs, relevées seulement dans les 159 ha centraux, regroupent, pour leur part, en grande majorité, une à quatre structures $(84 \%)$. La distance séparant les petites unités est au minimum de 25-30 m. Rappelons qu'à toutes les époques l'habitat maya de base comporte le plus souvent plusieurs bâtiments (dont une cuisine) groupés autour d'un espace commun.

Une cinquantaine d'édifices multi-pièces, assez bien conservés, constitue notre échantillon de l'architecture Río Bec la plus élaborée. Le plan tripartite y est courant : un corps central, en général d'au moins deux pièces disposées l'une derrière l'autre (pièces dites « en tandem »), est encadré par deux ailes latérales comportant, chacune, deux pièces ou plus. Les murs sont faits d'un noyau de tout-venant enserré entre deux parements de pierre de taille, de modules et de qualités variables, mais souvent assez grands (carrés de $40 \mathrm{~cm}$ de côté) et d'excellent fini de surface à l'extérieur, plus petits et de qualité moindre à l'intérieur (Figure $4 \mathrm{a}, \mathrm{b}$ ). Les toits étaient maçonnés en voûte à encorbellement et les surfaces, stuquées et peintes. Les mortiers de chaux d'excellente qualité (Gillot 2014) ont assuré une conservation relativement bonne depuis l'abandon $\mathrm{du} \mathrm{x}^{\mathrm{e}}$ siècle, dans une zone où la forêt s'est rapidement reconstituée pour n'être plus jamais colonisée. Même très récemment, l'installation de colons mayas yucatèques à une douzaine de kilomètres au nord de la zone nucléaire du projet n'a pas entraîné de mises en cultures dans la zone d'étude, la micro-région faisant partie de la réserve de bois et de gibier du village (20 de Noviembre). La bonne visibilité des vestiges du système agricole s'explique aussi par cette absence de mise en exploitation. De grands incendies naturels ont cependant affecté périodiquement la forêt et les bâtiments.

L'architecture Río Bec, comme celle des autres styles mayas classiques, se caractérise donc avant tout par la pierre de taille et les mortiers de chaux. 


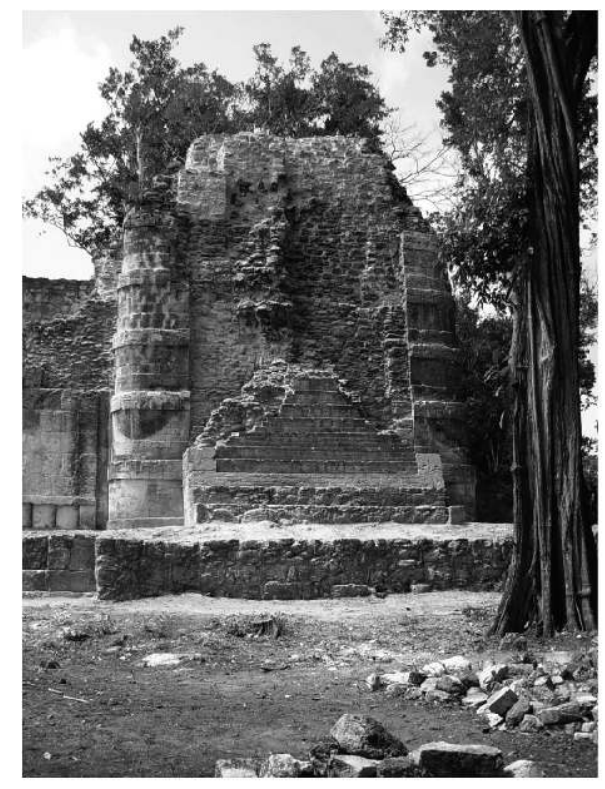

a

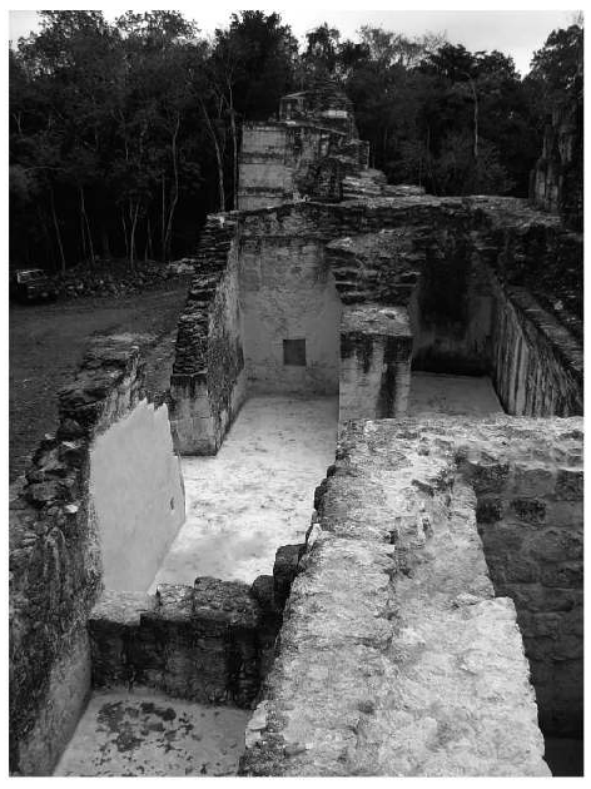

b

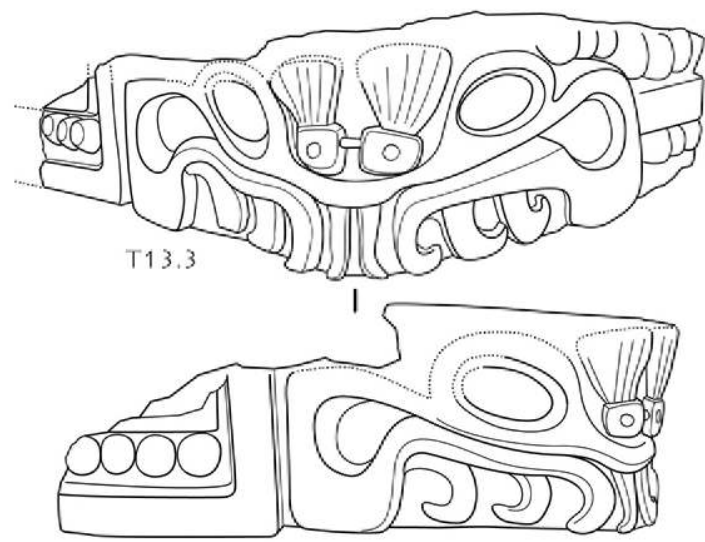

C

FIG. 4 - La structure principale du Groupe A (5N2) ; a : sur la gauche du cliché, parements externes au contact de la tour N-O ; b : parements internes ; $\mathrm{c}$ : motif du monstre de la Terre qui décorait la moulure supérieure du bâtiment en façade principale (nord).

Dessin : Nicolas Latsano poulos. 
Les ressources élémentaires en sont le bois de combustible et le calcaire local, une pierre tendre extraite dans des carrières superficielles, encore bien visibles, souvent voisines des plus grands édifices et occupant des espaces assez considérables au milieu des vestiges agricoles (voir Figure 5, notamment le secteur au sud du Groupe D ou celui à l'ouest du Groupe R ; Eaton 1974 ; Turner 1983 ; Gillot en préparation). En d'autres termes, les deux composantes fossiles résultant de ce qui fut sans doute le groupe d'activités principales de la société Río Bec, à savoir la construction de résidences monumentales et l'exploitation agricole des terroirs locaux, sont fortement imbriquées spatialement.

L'architecture de pierre taillée Río Bec est différente de celle du Petén, des Chenes et du Puuc: en ce qui concerne son registre fonctionnel, elle se limite strictement à l'univers résidentiel et domestique. Pour la période correspondant au style Río Bec bien formé, aucun temple, aucune pyramide véritable et aucun terrain de jeu de balle ne sont connus (voir Taladoire ce volume). À l'exception de quelques bâtiments spécialisés à grandes salles de réunion, tous les édifices multi-pièces de style Río Bec étaient des résidences. Chaque pièce dotée d'une grande banquette maçonnée ou plutôt, et dans bien des cas, chaque paire de pièces « en tandem » servait de logement à une famille nucléaire; les pièces plus grandes $\left(28 \mathrm{~m}^{2}\right.$ et plus) servaient aussi de salles de réception et de réunion et les pièces plus petites $\left(7-11 \mathrm{~m}^{2}\right)$, dénuées de banquette, étaient peut-être utilisées pour le stockage de denrées volumineuses. Dix-huit maisons ont été entièrement fouillées, révélant de nombreux détails comme des accroche-rideaux associées aux portes, des niches sous les banquettes et dans les murs, des dispositifs divers d'étagères aux murs et sous les voûtes (Arnauld et al. 2013 ; Michelet et al. 2013). Nos fouilles ont en outre permis d'identifier cinq cuisines collectives partagées par plusieurs logements: ce sont des constructions séparées, non voûtées (Sion 2010 ; Déodat et Arnauld 2012). Des décharges de matériel céramique ont été découvertes aux abords des maisons. Mais le mobilier trouvé in situ sur les sols intérieurs est extrêmement rare, sauf dans les cuisines.

La diversité des unités d'habitat en termes de configurations spatiales (plusieurs maisons autour d'un patio, ou bien alignées, ou formant un ensemble compact : Nondédéo et al. 2013) et de qualités de maçonnerie (décors de mosaïques de pierre, matériaux plus ou moins bien taillés, voûtes plus ou moins élaborées: Nondédéo et Patrois 2010) est néanmoins marquée par une forte continuité depuis les petites maisons simplement maçonnées jusqu'aux très grandes résidences, dont les façades sont parfois flanquées de tours imitant des temples sur pyramides (Michelet et al. 2013). Il n'est pas facile de définir les catégories d'une hiérarchie, tant la gradation est progressive et les différences subtilement signalées (Nondédéo et al. 2013 ; voir aussi Marcus 2004). Plusieurs différences ont d'ailleurs une signification temporelle, les maisons les plus élaborées ayant des séquences de construction assez longues. Dans certains cas, il est clair que les travaux d'agrandissement étaient programmés longtemps à l'avance 


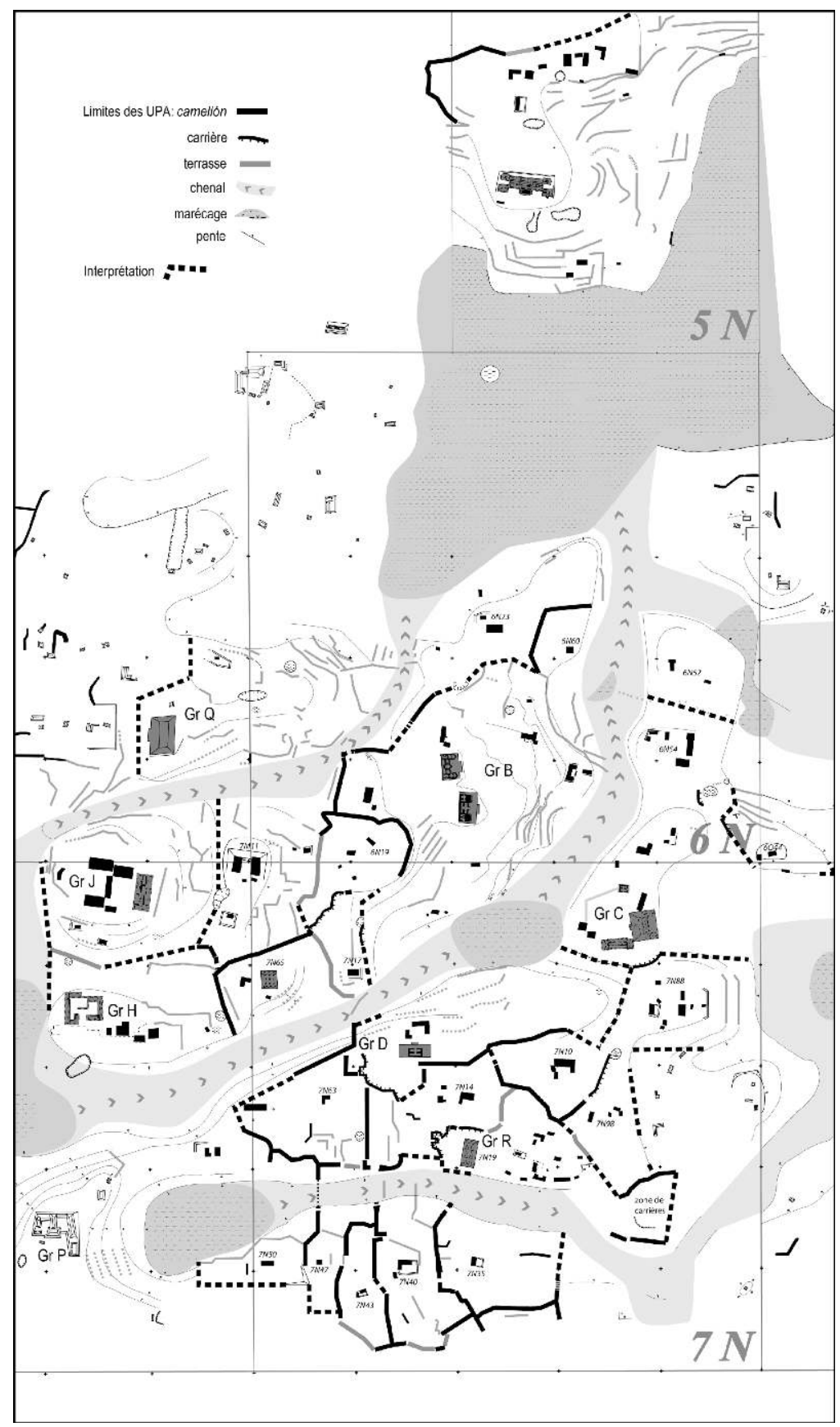

FIG. 5 - Délimitation des unités de production agricole (UPA) dans une partie de la zone nucléaire de Río Bec (dessin : Boris Vannière et Éva Lemonnier). 
mais n'ont jamais été faits. Les décors des façades répètent à l'infini des motifs liés au monstre de la Terre, entité surnaturelle, rarement des motifs anthropomorphes (Nondédéo et Patrois 2007) : Figure 4c. À l'intérieur même d'une seule grande résidence, des différences de hauteurs de sols et d'escaliers d'accès suggèrent que les habitants attachaient une grande importance aux distinctions sociales de tous ordres. Mais c'est toujours la salle arrière du corps central qui a le sol le plus haut. Des données iconographiques indépendantes (récipients céramiques du Classique récent à figures humaines, graffiti sur les murs) aident à interpréter cette configuration particulière comme reflétant le rôle premier du chef de famille qui logeait dans cette salle, où il recevait les visiteurs étrangers (Arnauld et Michelet 2010 ; Arnauld et al. 2013). Il y a peu de doute sur le fait que la hiérarchie complexe reflétée par les différences architecturales entre les maisons avait une signification en partie chronologique, en partie sociale et, pour les plus grands groupes, politique.

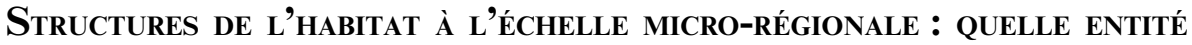 POLITIQUE?}

Contrairement à d'autres établissements mayas classiques - y compris plusieurs de la région Río Bec même -, le site éponyme est très vaste $\left(24 \mathrm{~km}^{2}\right.$ d'après la reconnaissance des $100 \mathrm{~km}^{2}$ ) et ne comporte pas de centre nucléé. Aucun processus d'agglomération n'a eu lieu localement au temps de l'apogée ${ }^{1}$, les unités d'habitat étant assez régulièrement espacées et les vestiges du système agricole imbriqués entre celles-ci. Toutes ces unités sont de fonctions résidentielle et domestique, ce qui n'exclut pas que, comme on l'a mentionné, certains groupes monumentaux aient disposé de salles de réunion. A priori donc, Río Bec est un site relativement amorphe sur le plan de la structure politique.

Trois ensembles de données sont cependant pertinents concernant l'organisation politique et doivent être discutés. Premièrement, la majorité des 73 groupes monumentaux inventoriés forme deux grandes concentrations spatiales, l'une située au nord-est, l'autre au centre (Figure 2). La concentration centrale compte 45 groupes, celle du nord-est 26 - deux groupes, Omelita et El Porvenir, étant isolés au sud-est, hors micro-région (Nondédéo et al. 2013). La distance minimale entre les deux concentrations est d'un kilomètre. Mais cette apparente partition/structuration micro-régionale pourrait être en rapport principalement avec un drainage naturel qui forme, entre les deux ensembles, une large entaille selon un axe SO-NE, cela dans une pente générale descendant vers l'est.

Deuxièmement, trois groupes monumentaux sur les 73 présentent une configuration un peu différente des autres, en vraie(s) place(s) entourée(s) de quelques édifices publics incluant temple-pyramide et/ou terrain de jeu de balle, tous construits avant l'apogée Río Bec. Il s'agit de Kajtún (62 structures) qui remonte, 
en majeure partie, au Préclassique récent (200 av. J.-C.-200 apr. J.-C.), du Groupe II (8 structures), daté du Classique ancien (200-600 apr. J.-C.), et du Groupe V (18 structures), correspondant un peu au Classique ancien, d'un côté, et surtout au Classique terminal (800-950 apr. J.-C.), de l'autre. Ces différents groupes montrent en fait une composition et une configuration plus typiques de la tradition du Petén que celle de la région Río Bec (Nondédéo et Lacadena 2004). Kajtún, par exemple, qui a rassemblé avec le groupe voisin Dzibil un assez grand nombre de structures avant l'apogée Río Bec, aurait pu constituer le noyau d'une agglomération du type des cités du Petén, mais il a été en majeure partie abandonné ensuite. On doit aussi remarquer que les groupes en question sont nettement distants les uns des autres : Kajtún est situé dans la concentration nord-est, le Groupe II au sud, en dehors de la concentration centrale, de même que le Groupe V, localisé lui au nord de celle-ci. S'ils ont joué un rôle politique, ç’aurait été successivement : Kajtún a été le premier, au nordest, durant le Préclassique ; puis le Groupe II au sud-ouest aurait bénéficié du déplacement d'un éventuel pouvoir politique au Classique ancien; enfin, le Groupe V aurait constitué le dernier centre, au nord-ouest. Lui-même et le Groupe II n'ont cependant pas formé d'agglomération.

Troisièmement, c'est uniquement dans ces trois groupes à places publiques que se distribuent les 18 stèles répertoriées à Río Bec, dont 11 portent des inscriptions glyphiques et des figures humaines sculptées (Lacadena 2007; Nondédéo et Lacadena 2004 ; Taladoire ce volume). Ce type de monument est, dans l'aire maya, caractéristique du pouvoir royal, que la stèle marque dans l'espace, puisqu'elle est érigée en général sur la place publique, et dans le temps, puisque l'inscription qu'elle porte inclut le plus souvent une date calendaire. Sept dates ont pu être déchiffrées, échelonnées de 475 à 869 apr. J.-C., ainsi qu'un possible même titre royal ${ }^{2}$ (B'olonil ajaw, « roi de B'olonil») sur trois des monuments. Chacun de ces trois monuments a été érigé dans l'un des trois lieux, mais à des dates inconnues sauf la stèle 3 du Groupe V (9.9.0.0.0 ?, soit 613 apr. J.-C. : voir Lacadena 2007). L’iconographie des stèles, lorsqu'elle est conservée, s'inscrit tout à fait dans la tradition du pouvoir royal du Petén. Si les trois sites à stèles paraissent indiquer l'existence d'un pouvoir politique hiérarchiquement supérieur, celui-ci aurait dû être successif entre les trois lieux puisque c'est le même titre qui semble avoir été utilisé. Pourtant, plusieurs problèmes demeurent, au premier rang desquels les dates des stèles, lesquelles ne correspondent pas à celles de la construction et de l'occupation des groupes : ainsi, les stèles datées de Kajtún et du Groupe II, sauf une, sont postérieures aux phases de construction et d'occupation de ces mêmes groupes. Ce décalage suggère que la pratique de l'érection de stèles à inscriptions aurait eu à Río Bec une valeur à part et sans doute pas de portée communautaire. Les trois centres n'eurent d'ailleurs pas le monopole des inscriptions politiques puisque un texte comportant une date (805 apr. J.-C.) et deux noms a été retrouvé, non pas sculpté mais 
peint, sur les bordures des banquettes du corps central de la grande résidence $6 \mathrm{~N} 2 \mathrm{du}$ Groupe B au beau milieu de la concentration centrale (Arnauld et Lacadena 2004).

Les trois groupes à place publique ont été sondés, mais pas fouillés. Leur séquence est grosso modo connue, mais pas les activités dont ils auraient eu l'exclusivité, non plus que les relations qu'ils auraient pu entretenir avec les grandes capitales voisines, celles du royaume de Kaan tout particulièrement, Dzibanché à l'est, Calakmul à l'ouest, situées en gros à égale distance, environ $60 \mathrm{~km}$ à vol d'oiseau (Martin 2005; Nalda et Campaña V. 1998 ; voir aussi Nalda et Balanzario ce volume). Il est donc difficile d'affirmer qu'une entité politique unifiée ait vraiment existé dans ce secteur de Río Bec. Dernière inconnue politique de taille, la cité particulièrement nucléée et compacte de Becán est à moins de $30 \mathrm{~km}$ au nord-ouest de la micro-région étudiée, et c'est elle qui manifeste le plus d'innovations de style Río Bec dès la fin du Classique ancien, vers 500600 apr. J.-C. (Ball 1977 ; Eaton 1974 ; Nondédéo et al. 2010 ; Thomas 1981 ; Webster 1976). La conclusion la plus prudente qui s'impose est donc que la micro-région de Río Bec représentait, non pas une entité politique définie, mais une région rurale que se partageaient de nombreux groupes sociaux vivant en bon voisinage (aucune donnée n'indique en effet qu'un état de guerre ait prévalu). Ceux-ci s'étaient dotés d'institutions politiques minimales, parées, dans trois petits centres successifs et à certains moments, des attributs symboliques de la royauté traditionnelle au Petén. Ces trois centres n'auraient même pas eu le monopole des inscriptions politiques comme on l'a vu, ni sans doute celui des relations avec les royaumes voisins. Dans ces derniers, la diffusion rapide du style des résidences Río Bec après 750 apr. J.-C. (sans doute autant à partir de Becán que de Río Bec même) pourrait refléter l'extension de systèmes d'alliances entre groupes sociaux relativement autonomes. Ainsi, Río Bec représente bien un ensemble politiquement assez amorphe, sans préjuger cependant d'une évolution qui s'est peut-être dessinée à date très tardive (Nondédéo et al. 2013 ; voir aussi la note 1).

\section{Chronologie : Le TeMPS long en zone RuRale}

Quoiqu'elle soit dénuée de ces grands marais permanents qui ont fixé l'occupation des sociétés mayas d'agriculteurs au Préclassique (à partir de 1000 av. J.-C. : voir Pope et Dahlin 1989), la micro-région Río Bec ne fut pas colonisée beaucoup plus tardivement que les autres. Bien que nos fouilles n'aient pas eu pour objectif premier de les localiser, les témoignages céramiques de la deuxième partie du Préclassique moyen (600-200 av. J.-C., Figure 6) ne sont pas rares dans les 169 sondages effectués (Taladoire 2007a). Le nombre des unités d'habitat a ensuite augmenté du Préclassique récent au Classique ancien, mais les données de 


\begin{tabular}{|c|c|c|c|}
\hline Time Period & $\begin{array}{c}\text { Becan } \\
\text { Sequence } \\
\text { Ball } 1977\end{array}$ & $\begin{array}{c}\text { Río Bec } \\
\text { Ceramic } \\
\text { Complexes }\end{array}$ & $\begin{array}{c}\text { Diagnostic Types, Varieties } \\
\text { (v.) or Wares }\end{array}$ \\
\hline \multirow[b]{3}{*}{ TERMINAL CLASSIC } & \multirow{3}{*}{$\begin{array}{l}1050 \\
\text { Xcocom }\end{array}$} & $\begin{array}{l}1000 ? \\
\quad \text { XPUIIUK } 3 \\
950\end{array}$ & Tohil Plumbate \\
\hline & & $\begin{array}{l}\text { XPUHUK } 2 \\
900\end{array}$ & Fine Orange (Altar Group) \\
\hline & & $\begin{array}{l}\text { XPUHUK } 1 \\
850\end{array}$ & $\begin{array}{l}\text { Encanto: Yokat v. } \\
\text { Traino: Lodo } v . \\
\text { Tinaja Red: Tinaja v. } \\
\text { Yucatan Slate Ware }\end{array}$ \\
\hline \multirow{5}{*}{ LATE CLASSIC } & $\underline{830}$ & $\begin{array}{l}\text { MAKAN } 2 \\
790\end{array}$ & $\begin{array}{l}\text { Tinaja Red: Nanzal V. } \\
\text { Carro Modeled } \\
\text { Torro Gouged-Incised }\end{array}$ \\
\hline & $\begin{array}{l}\text { Chintok } \\
730 \\
\end{array}$ & MAKAN 1 & $\begin{array}{l}\text { Encanto: Pepino v. } \\
\text { Traino: Traino v. } \\
\text { Achote Black }\end{array}$ \\
\hline & \multirow[b]{4}{*}{$\begin{array}{l}\text { Sabucan } \\
450 \\
\end{array}$} & 700 & Ulil Fluted \\
\hline & & $\begin{array}{l}\text { KANLOL } 2 \\
625\end{array}$ & $\begin{array}{l}\text { Encanto: Alambre v. } \\
\text { Molino: Buitre v. } \\
\text { Becanchen Brown } \\
\text { Corona Red } \\
\end{array}$ \\
\hline & & $\begin{array}{l}\text { KANLOL } 1 \\
550\end{array}$ & $\begin{array}{l}\text { Molino: Buitre v. } \\
\text { Triunfo: Acahual v. } \\
\text { Becanchen Brown } \\
\text { Corona Red }\end{array}$ \\
\hline \multirow{3}{*}{ FARIYY CI.ASSIC } & & & $\begin{array}{l}\text { Triunfo: Acahual v. } \\
\text { Tacopate: Gremio v. } \\
\text { Aguila: Flamboyan v. }\end{array}$ \\
\hline & \multirow[b]{2}{*}{ Chacsik } & 425 & langostino Red: l,angostino $v$. \\
\hline & & $\begin{array}{l}\text { GUAYACAN } \\
\text { AD } 300\end{array}$ & $\begin{array}{l}\text { Aguila Orange: Aguilav. } \\
\text { Triunfo: Acahual v. } \\
\text { Tacopate: Tacopate v. } \\
\text { Dos Arroyos } \\
\text { Balanza Black }\end{array}$ \\
\hline LATE PRECLASSIC & Pakluum & $\begin{array}{l}\text { BOHOM } \\
300 \mathrm{BC}\end{array}$ & $\begin{array}{l}\text { Sapote Striated: Rastro v. } \\
\text { Sierra Red } \\
\text { Polvero Black } \\
\text { Flor Cream } \\
\text { Zapatista Trickle-on-Cream-Brown }\end{array}$ \\
\hline MIDDLE PRECLASSIC. & Acachen & $\begin{array}{l}\text { Undefined } \\
600 \mathrm{BC}\end{array}$ & $\begin{array}{l}\text { Sapote Striated: Anejo v. } \\
\text { Juventud Red } \\
\text { Chunhinta Black } \\
\text { Pital Cream }\end{array}$ \\
\hline
\end{tabular}

Fig. 6 - Phases et complexes céramiques à Río Bec avec mention des catégories représentatives de chaque période (d'après Sara Dzul). La plupart des types avaient été établis par Ball (1977) à Becán. 
la zone nucléaire des 159 ha, plus précises concernant les petites unités, indiquent que beaucoup ont été abandonnées entre la fin du Classique ancien et le début du Classique récent.

Pour 22 unités fondées et occupées avant 625 apr. J.-C., seulement 14 sont fondées en phase Kanlol 2 entre 625 et 700, puis 7 au Classique récent en Makan 1 (700-790 apr. J.-C.) et, finalement, 5 en Makan 2 entre 790 et 850. En réalité, la tendance à un dynamisme décroissant s'est accompagnée de l'abandon de nombreux habitats anciens. La prospection et le relevé micro-topographique ont révélé en effet l'existence de nombreux petits monticules de pierres (153 sur 501 structures d'habitat, $30 \%$ ), soit isolés, soit groupés par deux, dont la fouille par sondages (16 d'entre eux) a montré, tantôt qu'ils datent d'avant 650, tantôt qu'ils couvrent des vestiges d'habitats antérieurs à 650 apr. J.-C. Si on extrapole les datations obtenues en fouille à l'ensemble des unités prospectées, la diminution de l'occupation en nombre d'unités d'habitat est très nette en phase Kanlol, c'est-à-dire juste avant l'apogée Río Bec: un grand nombre de petites unités d'habitation ont été abandonnées au profit d'unités moyennes et grandes de maisons maçonnées logeant plus de familles regroupées. L'habitat Río Bec ne s'est pas densifié stricto sensu (au sens de « multiplié »), tout au contraire ; il s'est plutôt contracté et transformé en unités comportant des résidences plus grandes pouvant loger chacune plusieurs familles, et cela entre la fin du Classique ancien et le milieu du Classique récent. Cela ne signifie en aucun cas un abandon de l'habitat Río Bec, mais une transformation des structures d'implantation.

Pour cette période d'apogée dans toutes les Basses Terres mayas, contraction et fléchissement démographique (en quantité d'unités d'habitat) ne sont pas des processus qu'on signale pour les sites où des programmes amples de datation des zones résidentielles ont été menés. On parle, bien au contraire, d'augmentation de la population locale par urbanisation et immigration au Classique récent (Culbert et Rice 1990 ; Inomata 2004). Et c'est à ce moment que, partout, ont été entreprises les constructions de groupes monumentaux de palais où résidait l'élite (à partir de 600 apr. J.-C.). Sur ce point, Río Bec n'échappe pas à la règle puisque les débuts du style architectural Río Bec sont maintenant fermement datés de la phase Kanlol 1 (550-625 apr. J.-C. : Taladoire et al. 2013), avec un apogée tout à fait remarquable pour ses très grands édifices à tours et portes zoomorphes et pour leur date de construction vers 800-850 apr. J.-C. : Groupe I, structure XI et XV-XVII ; Groupe V, structure V ; Omelita, structure 1 ; Groupe IV, structure 2 ; Groupe E, structure 1; Groupe M, structure 1; Ceibarico A, structure 1; Groupe B, structure 6N1 et Groupe A, structure 5N2 (Nondédéo et Dzul 2010 ; Nondédéo et al. 2010 ; Taladoire et al. 2013, figures 6 et 8). Il y a donc, apparemment, contradiction partielle entre le développement de programmes de construction de grands édifices et la diminution du nombre d'unités d'habitat dans la zone nucléaire puisqu'un moins grand nombre de groupes résidentiels modestes aurait dû assurer la construction des grandes maisons à pièces multiples (voir Ball 2014 
qui parle à présent d'un abandon temporaire du centre de Becán et d'un hiatus d'occupation du lieu entre les phases Bejuco et Chintok/Xcocom, soit, selon lui, entre en gros 760 et 810 apr. J.-C.).

Deux solutions à cette contradiction sont envisageables : soit une redistribution complète de l'habitat se serait produite entre 550 et 650 apr. J.-C., agglomérant les unités petites autour des grandes unités à construire, soit la construction des grands édifices aurait été réalisée par une main-d'œuvre locale peu nombreuse, mais de façon progressive.

Sur la première hypothèse, agglomération de population locale autour de chaque nouvelle unité monumentale, les données des structures de l'habitat pour les phases Iximché-Kanlol-Makan (425-850 apr. J.-C.) sont négatives (Arnauld et al. 2012). Dans trois cas, toutefois, on repère la concentration d'une quinzaine de petites structures reflétant une nette discontinuité entre grands et petits logements (Groupes A-G, Q et R), ce qui demeure malgré tout limité pour parler d'une structure générale en quartiers hiérarchisés en interne. De réelles agglomérations se sont formées avant (à Kajtún-Dzibil) et après (Groupe I et OmelitaEl Porvenir, Figure 2), mais peu pendant la période 425-850 apr. J.-C.

$\mathrm{Au}$ Classique récent, la tendance fut plutôt à la formation assez généralisée - sauf au nord-est de la zone nucléaire, voir ci-dessous - d'un habitat maçonné de grandes dimensions avec peu d'habitats moyens alentour remplaçant l'habitat antérieur fait d'un grand nombre de petites unités anciennes. Ces dernières furent abandonnées et couvertes de pierres, les habitants ayant été expulsées vers (ou absorbées dans) les nouvelles unités plus grandes. On suppose que, simultanément, leurs terres ont été regroupées avec celles des grandes maisons. Dans les zones basses humides du quadrant nord-est de la zone nucléaire, qui sont, sur leurs bordures au moins, les plus favorables à l'agriculture, l'occupation apparaît à peu près nulle au Classique récent (Taladoire 2007b), ce qui autorisait leur exploitation agricole par les grandes unités adjacentes (Groupes A, B et C). En fait, la contraction de l'habitat paraît avoir eu une dimension à la fois résidentielle et agraire, constituant une sorte de " remembrement » des unités d'habitat et de production.

L'exploitation intensive des terroirs locaux agrandis et fortement aménagés demandait autant ou plus de main-d'œuvre que la construction des grandes maisons. Les groupes sociaux les plus ambitieux avaient tout intérêt à privilégier la coopération avec leurs voisins : les familles auraient été absorbées dans les grandes résidences et les unités moyennes. Le processus de contraction de la fin du Classique ancien a donc été d'ordre agricole et foncier plus qu'urbain, s'agissant de former des domaines agricoles en rapport avec de grandes maisons plutôt que des quartiers d'habitation (Arnauld et al. 2012). La corrélation positive obtenue entre le rang des maisons construites et la surface des unités cultivées environnantes le montre bien d'ailleurs, outre le fait que ces grandes unités (en réalité, de quelques hectares seulement) sont les plus densément terrassées (Lemonnier et 
Vannière 2007, 2013, table 2). En bref, les données invalident la première hypothèse, celle de la formation de quartiers nucléés de petites entités regroupées assurant la construction des grandes maisons (sauf trois cas). Les domaines agricoles formés disposaient, eux, d'une main-d'œuvre regroupée dans les résidences moyennes et grandes des nouvelles unités.

Sur la seconde hypothèse, c'est-à-dire la construction progressive dans le temps et par peu de main-d'œuvre locale, c'est ici encore le changement diachronique qui est le plus parlant. On a sérié les collections céramiques de 18 structures fouillées ou sondées formant les 9 unités du Groupe B (Figure 3 : Arnauld et al. 2007, 2010 ; Arnauld 2011) et, en une sériation distincte, les 13 structures formant les 8 unités du Groupe $\mathrm{D}$, le tout réparti spatialement sur un secteur de 18 ha et hiérarchiquement dans les 5 rangs définis pour l'habitat des 159 ha (Figure 3 : Nondédéo et al. 2013). On constate que trois unités du Groupe B sont abandonnées avant la construction des plus grandes maisons (6N1 et 6N2), une pendant et cinq peu après. Du côté du Groupe D, quatre unités le sont avant la construction de l'unité la plus monumentale et deux juste après. Les épisodes de construction de résidences maçonnées se sont succédées dans le temps de telle sorte que l'entraide entre voisins était possible. Et les datations céramique et radiocarbone des remblais des différentes phases techniques de construction (outre, dans un cas, la date calendaire maya peinte sur une banquette de la structure 6N2) indiquent que ces constructions ont duré jusqu'à 100 ans et plus (voir aussi Michelet et al. $2013^{3}$ ). Dans ces conditions, une main-d'œuvre locale peu nombreuse suffisait à la tâche (Adams 1998). Le modèle de l'autoconstruction lente, génération après génération, serait confirmé encore par le nombre non négligeable de grandes structures Río Bec occupées sans être terminées. Certains détails techniques d'un même bâtiment méritent d'être étudiés à la lumière de cette hypothèse (différences de construction, murs construits par compartiments, non-imbrication des angles).

En résumé, les données chronologiques suggèrent que le phénomène du style architectural Río Bec aurait été produit, plutôt que par l'expulsion de familles des petites unités abandonnées vers 550-650 apr. J.-C., par un relogement de familles voisines alliées en maisons maçonnées, tantôt un peu plus grandes, tantôt beaucoup plus grandes. Chaque groupe ainsi formé, quoique peu nombreux, était capable de mener la construction des grandes maisons pendant un temps assez long, tout en assurant l'exploitation d'un terroir agrandi par le même processus. La coopération, plus ou moins forcée, aurait donc joué à plein à l'intérieur des entités formées autour de chaque groupe, ce qui pourrait expliquer la continuité observée dans la hiérarchie morphologique des résidences. En dépit de l'ampleur des constructions, dont beaucoup sont vraiment monumentales, la zone est restée rurale, avec une faible densité de population. 


\section{L'AUTONOMIE ÉCONOMIQUE DES DOMAINES}

La plupart des cités mayas classiques des Basses Terres ont une densité de l'ordre de deux à quatre unités d'habitat à l'hectare (Copán, Palenque et quelques autres en ont plus, près de leur centre: voir Drennan 1988; Lohse 2004, table 6.7 ; Arnauld et Michelet 2004 ; Arnauld 2008). À Río Bec, elle serait de 0,55 au Classique récent (86 unités datées Makan sur 159 ha: Nondédéo et al. 2013). C'est donc bien une densité faible de «zone rurale », même si les résidences grandes et moyennes étaient imitées de " palais » dont l'invention est peut-être à attribuer plutôt à la cité voisine de Becán, où la densité de l'habitat était bien supérieure, et cela non sans rapport sans doute avec l'existence d'un fossé périmétral ancien qui aurait « contraint » spatialement le développement de la cité (Webster 1976).

\section{Le contexte agricole}

Les façades prestigieuses à Río Bec étaient décorées de motifs qui évoquent le monstre de la Terre (Figure 4c) et cette iconographie est celle d'une forme de pouvoir et de prestige en partie différente des images du pouvoir politique royal présentes, en revanche, sur les stèles à inscriptions (roi debout sur un guerrier captif : Nondédéo et Patrois 2007). Il s'agit d'expressions de puissance et de prestige liées à l'agriculture et au contrôle de terres héritées par le biais des « maisons durables » (Beck 2007), c'est-à-dire entièrement maçonnées et, donc, fixant sur place les paysans auparavant plus mobiles du fait de la pratique d'une agriculture itinérante (voir plus avant ; Arnauld 2014). Plus proprement politiques, les figures humaines sont rares. Mais il en existait toutefois quelques-unes, par exemple sur la crête faîtière de la grande résidence $6 \mathrm{~N} 1 \mathrm{du}$ Groupe B, dont la voisine, $6 \mathrm{~N} 2$, comportait, elle, des inscriptions peintes sur ses banquettes et, sur les piliers supportant ces dernières, des bas-reliefs peints évoquant des scènes de cour. Ces éléments renforcent l'allure semi-politique de cette paire de résidences monumentales.

L'iconographie du monstre de la Terre a pris des proportions extraordinaires dans le cas des portes zoomorphes, cependant peu fréquentes à Río Bec même (Groupe I, structure XVII). Elles confèrent sans doute à la maison une dimension mythique et rituelle par laquelle les occupants apparaissaient tantôt ingérés par la gueule du monstre, tantôt expulsés de celle-ci - les dents étant figurées clairement sur les chambranles, le seuil et au-dessus du linteau de l'entrée centrale - et possédaient de la sorte, en tout état de cause, un accès à l'intérieur de la Terre (Baudez 2002). Le contexte rural et agraire de Río Bec, tel que récemment démontré par nos recherches, renforce le caractère tellurique et agraire à la fois de cette iconographie dont le propre serait d'assurer conjointement une emprise sur des terres et sur leur fertilité, en somme la richesse agricole. Dans le cas de la 


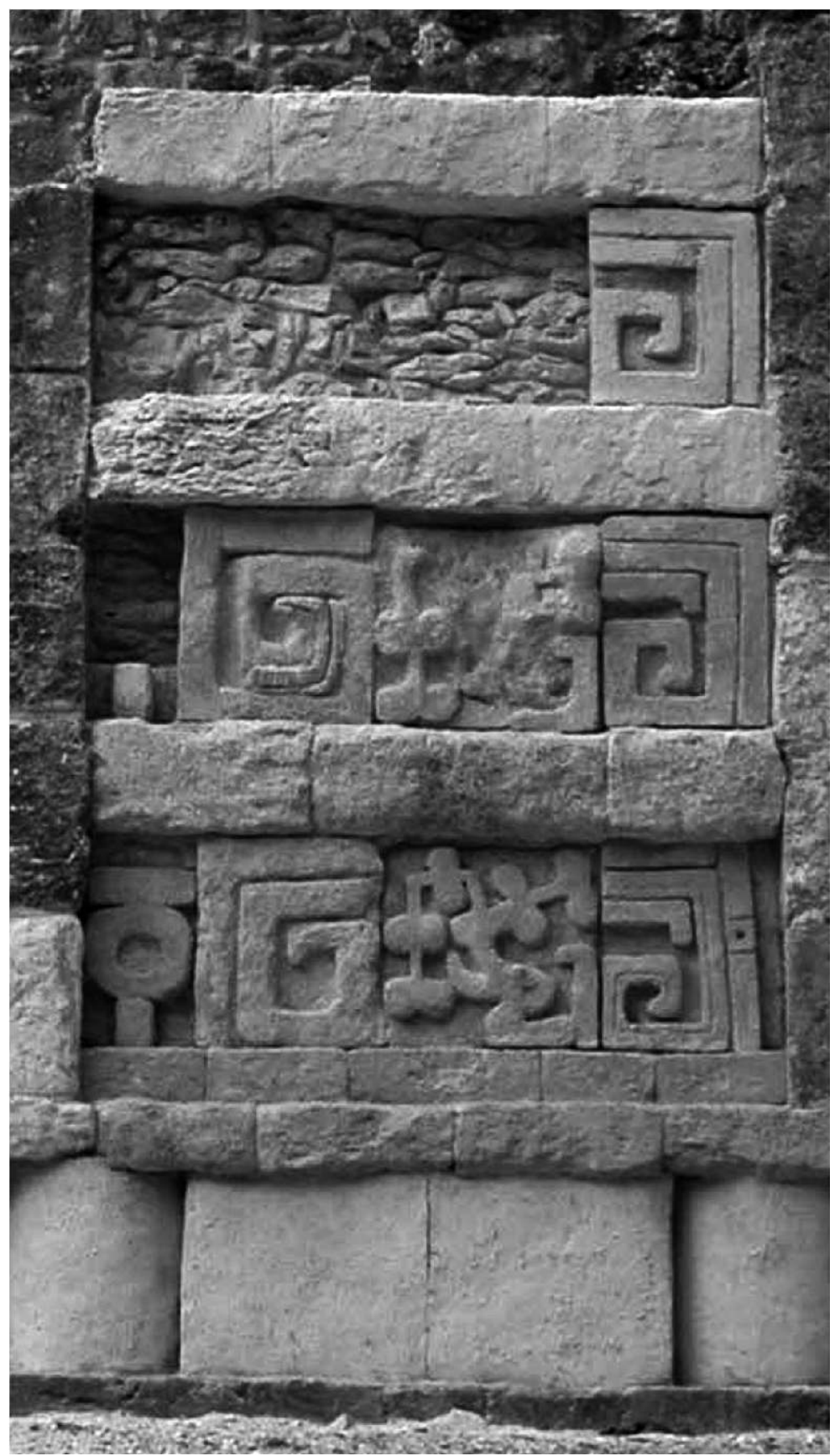

FIG. 7 - Plants de cacaoyer poussant dans la fente frontale du monstre de la Terre (façade principale de l'édifice 5N2 de Río Bec). 
façade de la très grande résidence du Groupe $A(5 \mathrm{~N} 2)$, des plantes sont montrées croissant dans la fente frontale entre les yeux géométriques du monstre qui représente la terre (Andrews 1995). Cette plante est répétée (sans doute 24 fois) sur la longue façade avec assez de cohérence pour qu'on y reconnaisse des arbustes de cacao, la richesse agricole par excellence des Mayas des Basses Terres (Figure 7 : Michelet et al. 2004a).

\section{Une agriculture intensive}

Point n'est besoin, cependant, de portes zoomorphes ou de façades monumentales pour établir l'autonomie agricole de l'unité d'habitat liée à l'exploitation par le groupe de co-résidence d'un domaine environnant, délimité. Comme on le verra, tous les vestiges archéologiques de la zone nucléaire de Río Bec trouvent leur cohérence dans l'interprétation qui en est faite d'une stricte superposition d'une unité d'exploitation agricole sur une unité d'habitat (farmstead et homestead comme l'avait déjà vu Eaton, 1974 ; voir aussi Dunning 2004), en dépit de dimensions très réduites en superficie. Le petit groupe de maisons est environné de parcelles aménagées et très vraisemblablement cultivées de façon intensive et continue (sans jachère ni brûlis). Il faut noter d'emblée que toute densification/multiplication des habitats aurait eu pour effet de réduire la surface des terres cultivables alentour. La stabilité relative (la non-croissance: voir ci-dessus) du maillage de l'habitat maintenait les dimensions des unités de production agricole. Mais, on l'a vu, l'abandon des petites unités anciennes aurait amplifié certains domaines, en particulier ceux des Groupes A, B et C, lesquels trouvaient en outre à s'étendre vers le nord-est dans les parties basses inoccupées à l'apogée Río Bec.

Grâce à un travail spécifique de relevé micro-topographique et de fouilles, le projet Río Bec a mis au jour et élucidé des structures agraires douées d'une grande homogénéité (Lemonnier et Vannière 2013). Autour des maisons plus ou moins maçonnées, certaines monumentales, d'autres plus modestes, des tas de pierres parsèment les espaces environnants, vestiges de petites maisons plus anciennes ainsi «marquées ", résultat d'épierrages et/ou modalité de protection d'arbres productifs. Le terrain est parfois terrassé, plus particulièrement à proximité des grandes maisons. Des levées de pierres - et terre - ou camellones, longues de dizaines de mètres délimitent les unités de production là où la topographie naturelle ne le fait pas clairement, peut-être en prévention de conflits d'ordre foncier (voir par exemple, sur la Figure 5, l'importante levée située à l'ouest-nordouest du Groupe A ; Lemonnier et Vannière 2013). Ces trois composantes - tas de pierres, terrasses et levées -, ont été repérées depuis longtemps dans la région Río Bec (Carrasco et al. 1986a ; Eaton 1974 ; Thomas 1981 ; Turner II 1983), mais leurs fonctionnalités et leur imbrication dans l'habitat hiérarchisé n'avaient pas été étudiées. 
L'archéologie et la sédimentologie donnent à voir et interprètent des structures au sol qu'on ne peut restituer sûrement qu'avec l'aide d'informations ethnohistoriques et ethnographiques. La pratique habituelle dans l'aire maya semble être l'exploitation agricole d'une zone par le groupe de la famille étendue, même si ce groupe tend, toujours et partout, à se subdiviser (Farriss 1984 ; Wilk 1988). Il n'y avait pas de travail salarié et le rôle des esclaves est considéré comme négligeable pour la période classique (du fait en particulier qu'aucun habitat spécifique ne le reflète). Au XVI siècle, les familles nobles yucatèques garantissaient l'étendue et les limites du territoire du groupe (se chargeant de résoudre les conflits) et, pour s'assurer l'accès à de bonnes terres, développaient des stratégies d'alliances (Okoshi 2006, 2012). Il n'y avait pas de notion de propriété du fond par opposition à l'usufruit et, par conséquent, il n'y avait pas de système de rente de la terre, de location ou de tribut versé contre l'usufruit (Earle 2000). Cependant, les aménagements réalisés sur une parcelle (arbres fruitiers, terrasses, clôtures) en assuraient la transmission aux enfants de celui qui les avait réalisés (McAnany 1995, pp. 69-99, 1998 ; Roys 1943, p. 28). En raison, soit de cette ancienne coutume, soit du respect dû au défricheur initial dont l'habitat très ancien (monticules archéologiques) est souvent visible dans la parcelle, le cultivateur maya assure, encore maintenant, sa fertilité par un échange rituel avec l'esprit de la terre outre, bien entendu, par son travail (Vapnarsky et Le Guen 2011). Il fait ainsi lui-même référence à la parcelle cultivée anciennement autour de la maison ruinée, tandis qu'aujourd'hui cette même parcelle peut se trouver en dehors de tout habitat moderne, loin de sa propre maison.

Les Mayas cultivaient, et cultivent encore, à la fois une parcelle en infield, près de la maison, et des parcelles en outfield, les milpas à proprement parler, où se pratique le système de la quema y roza (essartage-écobuage, brûlis sur abattis), à distance de la maison et en relative itinérance (Arnauld 2014; Killion 1992 ; Lemonnier 2009 ; Palerm et Wolf 1972 ; Stark et Ossaz 2007, p. 389). Au Yucatán, les Espagnols ont interdit toute culture du maïs à l'intérieur des villages (Okoshi 2006, p. 92 : ordonnance de López Medel), mais, dans les Hautes Terres, encore actuellement, les Mayas cultivent le maïs et d'autres plantes vivrières jusqu'aux murs des maisons (voir aussi les fouilles des extérieurs des maisons de Cerén : Sheets 1992). De nos jours, la milpa itinérante sur brûlis, en outfield, loin de la résidence, associée à la chasse, est de moins en moins pratiquée en raison (entre autres) de la colonisation générale des Basses Terres - les feux de forêt demeurant tout de même d'une fréquence remarquable aux mois d'avril-mai dans une bonne partie de la région.

De façon analogue, l'extension des zones habitées au Classique récent, que l'on constate notamment dans la région de Río Bec, laisse supposer que la production outfield n'était plus véritablement une option générale. Cette hypothèse, cruciale, n'a pas été pour l'instant rigoureusement vérifiée dans le secteur, mais la proximité des unités d'habitat et de production et leur extraordinaire 
extension sur la surface étudiée la rendent plausible. Si l'hypothèse est exacte, l'agriculture intensive pratiquée à l'intérieur des zones résidentielles devait donc assurer l'essentiel de la subsistance des co-résidents. Il est cependant possible que la zone inondable nord-est, proche des puissants Groupes A, B et C, ait été mise en culture au Classique récent en milpas itinérantes ou tournantes, sans compter la possible utilisation des secteurs de ce type comme réserves forestières, voire pour pratiquer des formes de sylviculture (Dussol 2012).

\section{Récoltes et grandes maisons}

Pour le Classique à Río Bec, l'unité de production correspondait donc à l'unité d'habitat et les pratiques agricoles avaient pour cadre social le groupe de parenté en co-résidence, doué d'une forte autonomie socio-économique et agraire. Groupe bien souvent petit si l'on en juge par la fréquence des unités d'habitat à deux structures, soit une seule famille nucléaire probablement. Le parcellaire était assez fixe, les maisons « durables » en assurant la transmission de génération en génération (voir Webster 2005). À vrai dire, chaque petit domaine était autant aménagé sur le plan agricole qu'il était marqué par les cicatrices de la construction résidentielle, carrières de pierres et tas de matériaux (Figure 5). C'est sur cette combinaison qu'il faut ici insister, le détail des pratiques agricoles, particulières aux conditions pédologiques et hydrologiques des très petites parcelles (moins d'un quart d'hectare), étant exposé ailleurs (Lemonnier et Vannière 2013). La corrélation, déjà évoquée, entre l'aménagement agricole (terrasses), la taille des unités de production en nombre d'hectares et le rang hiérarchique des maisons maçonnées (ibid., table 2) est un résultat d'analyse important. Il indique que la société locale investissait bien ses ressources dans la formation de domaines intensivement cultivés, sous le contrôle vraisemblable des grandes maisons, susceptibles, par leur prestige et leur capital agraire, d'attirer dans leurs murs les familles paysannes des alentours (logique des « agrandisseurs » de John Clark, voir Wells 2006, pp. 272-273). Du point de vue économique, le « phénomène Río $\mathrm{Bec} »$ tient dans cette gageure de la double production (agricole et résidentielle) et de l'attraction socio-économique, dans un équilibre précaire, qui a tout de même duré au moins de 700 à 950 apr. J.-C. pour certaines unités d'habitat.

Les deux projets étaient simultanés et imbriqués : les carrières de calcaire ont été laissées ouvertes au détriment des parcelles à cultiver, mais elles ont pu aussi, occasionnellement, être utilisées à la manière de pépinières; des matériaux de construction ont été, eux, accumulés autour des mêmes parcelles pour, selon les cas, les délimiter, les épierrer et/ou marquer l'habitat des ancêtres, tout en préparant la construction des remblais des nouvelles maisons. L'état d'aménagement des parcelles dans la zone nucléaire est remarquable et le tonnage de matériaux calcaires extraits, transportés, accumulés et maçonnés sur ces petits domaines serait impressionnant s'il pouvait être calculé. Chacun des groupes sociaux 
semble avoir profité d'une conjoncture pacifique, dans une relative indépendance politique vis-à-vis des deux grandes capitales de l'est et de l'ouest, Dzibanché et Calakmul, et compté sur un degré de coopération de la part de ses plus proches voisins, dans une période climatique sans doute assez favorable, au moins jusque vers 810 apr. J.-C.

En somme, ce que le bref apogée Río Bec signifie (en Makan et début Xpuhuk, de 700 à peut-être 900 apr. J.-C.), c'est une réelle prospérité pour des (petits) groupes de parenté de producteurs agricoles n'ayant pas d'autre ressource que la terre environnant leurs maisons et l'accès plausible à quelques zones basses inondables. Semble aussi avoir eu cours une ambition sociale et politique consistant à construire des maisons maçonnées de plus en plus grandes pour y mener des stratégies d'alliances (matrimoniales: Arnauld et al. 2013) de plus en plus lointaines et prestigieuses. L'association étroite, sur chaque petit domaine, des deux types d'activités signifiait un investissement important de la prospérité agricole dans la construction résidentielle.

Prospérité tardive et limitée, car l'archéologue constate que bien des maisons n'ont jamais été terminées, des pièces jamais aménagées. L'abandon, non pas sans doute de l'ensemble de la zone, mais d'une large partie des résidences, est intervenu probablement assez longtemps après la sécheresse du début du $\mathrm{IX}^{\mathrm{e}}$ siècle. D'ailleurs, l'usage tardif d'un réservoir d'eau dans la zone-test (datation Poz12419, $1000 \pm 30$ BP, 980-1060 cal yr AD, 2 sigma) et les restes de rituels effectués près de la grande maison du Groupe D tombant en ruines, sans oublier de très nombreux graffiti post-abandon (Patrois 2013), suggèrent que quelques habitants sont restés sur les terres, une fois désertées les maisons maçonnées coûteuses à construire et à entretenir.

\section{Artisanat et échanges}

Les paysans déserteurs n'ont rien laissé sur les sols stuqués de leurs résidences, rarement même les pierres à moudre dans les cuisines. À vrai dire, auparavant, ils n'avaient guère laissé de traces de leurs possessions mobilières : rares associations céramiques dans les sépultures (Pereira 2007, 2013), pauvres céramiques rituelles dans les quelques dépôts de fondation, aucune diversité de mobilier céramique ou lithique dans les dépotoirs (Andrieu 2007, 2009, 2013), absence quasi totale de céramique polychrome, de figurines, rares pesons à filer. La plupart des types céramiques, identifiés par la céramologue Sara Dzul et datés de la grande période Río Bec (550-950 apr. J.-C. ; Dzul, in Michelet et al. 2008), est d'origine locale ou d'une région proche. Les décapages des sols extérieurs n'ont révélé aucun atelier. Même les rares récipients de bonne qualité sont assez simples, comparés à leurs équivalents contemporains des Basses Terres centrales.

L'obsidienne est un bon marqueur de l'intensité des échanges à longues distances, puisque les sources les plus proches de ce verre volcanique se situent à 
plusieurs centaines de kilomètres du site. Or ce matériau est extrêmement rare à Río Bec, où il représente moins de $0,5 \%$ de la collection lithique, avec une fréquence de moins de 0,01 fragment par unité fouillée, l'une des plus faibles des Basses Terres (Andrieu 2009, p. 204). On constate toutefois un léger développement de l'importation de l'obsidienne au Classique terminal, bien que limité (ibid., p. 358). Cette période voit également l'augmentation de la présence d'un silex brun d'origine inconnue (Andrieu 2013, p. 29), exclusivement utilisé pour la fabrication de pointes de projectiles et feuilles lancéolées. La présence, en faible quantité, d'éclats diagnostiques de ces productions, régulièrement répartis dans l'ensemble des structures fouillées, indique que ces pièces bifaciales étaient très probablement produites par des tailleurs itinérants qui les échangeaient à mesure qu'ils les façonnaient sur place (ibid.). Ce modèle de production/distribution contraste nettement avec la fabrication de bifaces épais en matière première locale, de bonne facture, découverts en faibles quantités dans l'ensemble des groupes fouillés. Pour ces derniers, l'absence des éclats de production correspondants indique qu'ils n'étaient pas produits localement, mais plutôt sur les lieux d'acquisition/extraction de la matière première - pas forcément très lointains ou qu'ils étaient obtenus par le biais d'échanges. La régularité de la répartition de ces derniers outils, malgré un habitat très dispersé (Freidel 1981), est compatible avec une distribution de type « place de marché » (Hirth 2008). Enfin, la comparaison avec la distribution de la matière première utilisée pour ces mêmes outils à Calakmul montre que ces marchés ne diffusaient pas sur une distance supérieure à $60 \mathrm{~km}$ de diamètre (Andrieu 2013).

Ces quelques caractéristiques, à savoir la rareté de l'obsidienne, la présence d'un silex brun exogène, distribué par des tailleurs itinérants au Classique récent et terminal, et la possible distribution de certains outils bifaciaux par le biais de marchés temporaires locaux, ont également été observés à Calakmul et à Becán (Andrieu 2009, 2013 ; Braswell 2013 ; Rovner et Lewenstein 1997). À l'inverse, au-delà de la région, certains sites tels que Naachtun (Andrieu et Sion sous presse) présentent une structure et des réseaux d'approvisionnement tout à fait distincts. Les quelques caractéristiques citées montrent donc bien une spécificité de la région Calakmul-Becán-Río Bec au Classique récent-terminal. Ainsi, les données lithiques convergent avec les données céramiques et confirment l'existence d'une économie assez intégrée localement, mais en majeure partie coupée des réseaux d'échanges à longue distance.

Les enterrements comportent, pour leur part, un mobilier assez diversifié (Pereira 2007, 2013), mais qui reste d'assez pauvre qualité et dénué d'éléments importés, y compris dans le cas des sépultures d'ancêtres trouvées parfois en position axiale dans différents édifices, de haut rang notamment (voir le cas de la sépulture 8 dans la structure 7N1 du Groupe D). En fait, de rares céramiques de qualité supérieure ont été trouvées dans des résidences assez simples : voir la sépulture 6 dans la structure 7N4 du Groupe D : Figure 8. 


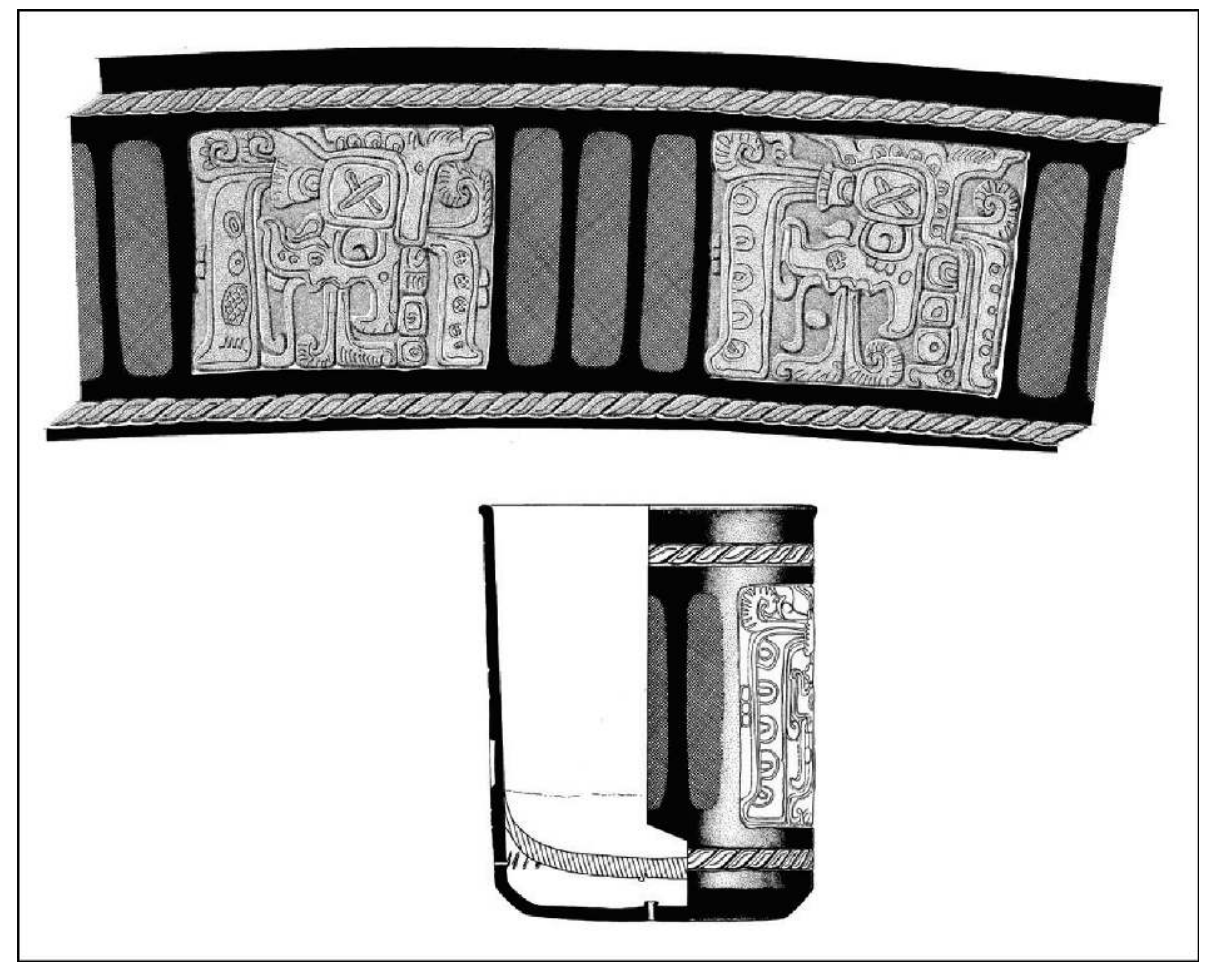

FIG. 8 - Vase cylindrique Torro Gubiado-Inciso (Gouged-Incised) provenant de la sépulture 6, structure 7N4 (dessin : Nicolas Latsanopoulos).

La culture matérielle associée aux résidences élitaires de Río Bec indique que, si leurs habitants « tenaient leur rang », ce n'était pas au moyen de luxueuses productions locales ou d'importations exotiques (sans préjuger évidemment d'éventuels produits périssables, tissus ou plumes notamment). Cette relative pauvreté mobilière est un argument de plus en faveur de l'indépendance politique de la micro-région Río Bec : a priori enclavée dans le royaume de Kaan entre Calakmul et Dzibanché et apparemment délaissée, elle est restée isolée des réseaux d'échanges (Figure 1; Arnauld et Nondédéo 2010; Nondédéo et al. 2010). Cela va bien de pair avec les investissements locaux dans les deux activités de l'agriculture et de l'architecture décrits plus haut, investissements qui auraient été exclusifs et se seraient faits au détriment des activités artisanales et commerciales.

La circulation, qui s'amorce tardivement dans la région Río Bec (Becán et les autres sites de style Río Bec inclus), de produits exotiques du type obsidienne, 
céramiques à pâte fine et céramique plumbate porte sur des quantités réduites. Il ne pouvait s'agir de « richesses », mais tout au plus de quoi assurer, par des échanges somptuaires, quelques alliances matrimoniales. Plus vraisemblable, dans cette perspective de l'acquisition de richesses, serait l'hypothèse que des denrées agricoles de valeur aient été localement produites, puis stockées, en particulier dans les plus petites pièces des grandes maisons (de moins de $11 \mathrm{~m}^{2}$ de surface). Sur certaines petites parcelles bien aménagées et bien entretenues aurait pu être récolté un coton ou un cacao local faisant concurrence aux productions de l'est des Basses Terres (Nord du Belize : voir Gómez-Pompa et al. 1990 ; Hammond 1978 ; Jones 1982 ; McAnany et al. 2002). Mais c'était là autant de surfaces déduites de la production des denrées alimentaires de base.

Tardivement, au Classique terminal, la remarquable expansion géographique du style architectural Río Bec, d'El Tigre à l'ouest jusqu'à Kohunlich à l'est, de Naachtun au sud jusqu'à Pechal au nord (Figure 1), suggère que les grandes familles de Río Bec avaient bien réussi à intégrer un réseau d'alliances et d'échanges propre au centre de la péninsule, cela après la fin de l'hégémonie politique et miltaire de Calakmul. Auparavant enclavée, cette société aurait finalement pu former, par le biais de ses investissements agricoles et architecturaux, des alliances lointaines, renforcées par l'échange de quelques produits exotiques et la fourniture d'hypothétiques denrées agricoles de valeur. Pour ces dernières, en l'absence de données paléobotaniques sur le site, seules l'ubiquité des images de la Terre et les représentations de probables cacaoyers sur la façade de la grande maison du Groupe A (Figure 7) fournissent des indices ${ }^{4}$.

\section{Discussion}

L'autonomie économique de chaque petit domaine trouve son illustration dans la grande majorité des résultats de recherche à Río Bec. Ce qui a été montré pour la production artisanale par Hirth (2008, p. 453) s'applique ici à l'agriculture (voir Lohse 2004). Les décisions liées à la production étaient prises au niveau de l'unité domestique. Si l'on voulait avoir une grande unité de production, il fallait parvenir à former une grande unité résidentielle regroupant des familles auparavant autonomes et le développement de l'architecture monumentale résidentielle aurait joué un grand rôle dans le processus.

En tout état de cause, l'indéniable diffusion du style architectural Río Bec dans presque tout le centre de la péninsule vers 800-900 apr. J.-C., reflète l'existence de réseaux socio-politiques et économiques tardivement formés. Outre donc la question, difficile à résoudre, des productions agricoles de valeur qui auraient pu servir de monnaie d'échange (coton, cacao ?), ces réseaux en posent deux autres : celle du rôle qu'y auraient joué les gouvernants des entités politiques régionales, et celle, plus économique, de la prospérité des groupes sociaux à 
Río Bec juste avant leur dislocation finale au cours du $\mathrm{x}^{\mathrm{e}}$ siècle, puisque les grandes maisons Río Bec ont partout été abandonnées, sauf à Becán. Sur la première question, on observera que la diffusion du style Río Bec à l'extérieur de sa région d'origine semble avoir concerné des complexes de résidences élitaires, mais pas des palais royaux qui, d'ailleurs n'existaient peut-être plus à cette époque : voir, par exemple, les trois résidences qui bordent le Patio D dans le Groupe Sud de Balamkú et qui paraissent bien copier des modèles Río Bec (Becquelin et al. 2005, figure 12). À Kohunlich, il semble que d'autres styles concurrents furent aussi adoptés dans une société locale éclectique, ouverte aux influences multiples et peu soumise à un pouvoir royal centralisé (voir Nalda et Balanzario ce volume). En somme, la diffusion du style architectural Río Bec mesurerait avant tout la faiblesse des rois dans tout le secteur (Fowler et Arnauld 2013 ; Nondédéo et al. 2010), surtout après la disparition de la dynastie Kaan à Calakmul. La profusion des résidences de style Río Bec refléterait donc bien une conjoncture politique, postérieure à 730 ou, mieux encore, à 800 apr. J.-C., dans laquelle les noblesses régionales déployaient leurs ambitions tandis que leurs gouvernants déclinaient, s'ils existaient encore.

Quant à la seconde question, prospérité des grandes maisons qui, pourtant, allaient bel et bien être abandonnées, on peut admettre qu'elle fut réelle même si l'inachèvement de plusieurs bâtiments en montre les limites. À quoi donc attribuer l'abandon progressif des grandes maisons au Classique terminal (à partir de 950 apr. J.-C.) à Río Bec (mais pas à Becán, l'une des rares cités mayas à survivre durant la période postclassique jusque vers 1100: Bueno 1999; Campaña et al. 2004) ? Le changement des routes d'échanges à longue distance, déplacées vers les côtes durant le Classique terminal (Turner II et Sabloff 2012 ; Dunning et al. 2012), s'il est acceptable dans le cas du probable centre commercial sans doute résilient que fut Becán, ne l'est guère pour la zone enclavée de Río Bec. Mieux vaut donc en rester au facteur de l'équilibre précaire que l'on suppose avoir existé entre production et construction, rendant les domaines peu résistants à la série d'années sèches qui se sont multipliées à partir de 810 et jusque vers 1100 apr. J.-C. (voir, notamment, Kennett et al. 2012) : abandonner les grandes maisons maçonnées permettait de revenir entièrement à la mobilité flexible et plus prudente des milpas itinérantes. Ce serait la fin d'un système de richesse reposant sur la terre et l'architecture maçonnée monumentale, la fin des pactes sociaux qui avaient cours dans cette société à maisons.

\section{Conclusion}

Le déclin assez rapide de Río Bec (autour de 950 apr. J.-C.) ne fut pas celui d'une royauté sacrée, bien faible ou même inexistante localement, ni celui d'un système où artisanat et échanges auraient tenu un grand rôle, puisqu'ils furent 
bien peu développés, non plus que celui d'une cité urbanisée. Quant au parcellaire aménagé, il pourrait, lui, ne pas avoir été totalement abandonné.

Ce que la société locale a abandonné, c'est l'habitat maçonné, de dimensions assez monumentales, coûteux à entretenir, et dont l'importance dans cette société du centre de la péninsule ressort à présent clairement. Les avantages des belles maisons, en termes d'alliances exogames prestigieuses qu'elles permettaient, diminuaient au fur et à mesure que les cités du centre de la péninsule se dépeuplaient et que les échanges changeaient de nature dans des royaumes fréquemment en guerre et avec des populations rendues à leur mobilité et à leur dispersion. Les cités de la côte du Nord du Belize, qui ont alors connu une prospérité commerciale indéniable, ont peu à peu fait évoluer leur architecture, abandonnant les voûtes maçonnées pour des toits plats à charpentes simples en bois. Les architectures Río Bec, Chenes et Puuc furent ainsi les dernières expressions d'un classicisme indéniable dans son élaboration somptueuse mais coûteuse, qui se prolongea jusqu'au tout début $\mathrm{du} \mathrm{XI}^{\mathrm{e}}$ siècle apr. J.-C. Après 1020, ni Chichén Itzá, ni, plus tard, Mayapan ne mirent en œuvre des édifices d'aussi bonne qualité. L'épisode de la pierre taillée n'avait été qu'un moment dans l'évolution socio-économique des Basses Terres, mais d'une visibilité exceptionnelle. *

* Manuscrit reçu en octobre 2014, accepté pour publication en décembre 2014.

\section{Notes}

1. Le Groupe El Porvenir, situé juste à l'extérieur et au sud-est de la micro-région d'étude (voir Figure 2) mais cependant topographié par le projet, constitue une exception en matière de concentration. Une exception, il faut le souligner, entièrement comprise chronologiquement dans le Classique terminal et représentant donc un phénomène tardif dans l'occupation du secteur.

2. En dehors de la stèle 1 de Kajtún-Dzibil, où il est complet, dans les deux autres cas (stèles $1 \mathrm{du}$ Groupe II et 3 du Groupe V), le titre en question est en partie illisible.

3. Dans le cas de la structure 5 N2, le plus gros bâtiment à tours de la zone des 159 ha, il a pu être montré que l'édification du bâtiment tel qu'on le voit aujourd'hui, à partir d'une unité initiale de deux pièces avait commencé vers 830-850 apr. J.-C. et n'était pas achevée à l'abandon du lieu autour de 950 apr. J.-C. Autrement dit, la construction de cette structure s'est étalée sur au moins cinq générations.

4. On notera toutefois que, parmi les charbons de bois provenant des sépultures 16 et 9 , dans un premier temps, du Theobroma sp. avait été suspecté (Dussol 2021). Les critères d'identification alors retenus n'étant pas suffisants pour certifier cette identification, ces charbons ont été finalement classés dans la catégorie des Indéterminés (Dussol et al. en préparation), mais l'hypothèse d'une culture locale du cacaoyer n'est pas fermée pour autant. 


\section{RÉFÉRENCES CITÉES}

ADAMs Richard E. W.

1998 «Structures as sites: the construction process and Maya architecture », in S. D. Houston (éd.), Function and meaning in Classic Maya architecture, Dumbarton Oaks, Washington (D.C.), pp. 123-140.

1981 «Settlement patterns of the Central Yucatan and Southern Campeche regions », in W. Ashmore (éd.), Lowland Maya settlement patterns, University of New Mexico Press, Albuquerque, pp. 211-257.

Adams Richard E. W., Richard C. Jones

1981 "Spatial patterns and regional growth among Classic Maya cities ", American Antiquity, 46 (2), pp. 301-322.

ANDREws George F.

1995 "Classic Puuc mosaic style architecture and geometric masks », Pyramids and palaces, monsters and masks. Vol. 1: Architecture of the Puuc region and the Northern plains areas, Labyrinthos, Lancaster (Cal.), pp. 169-197.

ANDRIEU Chloé

2007 «Una perspectiva regional de la economía lítica de Río Bec », communication présentée au symposium « Río Bec: génesis, desarrollo y percepción de un fenómeno cultural », P. Nondédéo (coord.), VIIo Congreso internacional de los mayistas, 8-14 de julio, Mérida.

2009 Outils mayas : distribution et production du silex et de l'obsidienne dans les Basses Terres, thèse de doctorat, préhistoire, université Paris Ouest Nanterre La Défense, 2 vol., 433 p., 109 p.

2013 «Late Classic Maya lithic production and exchange at Rio Bec and Calakmul, Mexico », Journal of field archaeology, 38 (1), pp. 21-37.

ANDRIEU Chloé, Julien Sion

Sous presse «En un cruce de camino: los intercambios en Naaachtun vistos desde el análisis del material lítico », in P. Nondédéo, D. Michelet, N. Grube (éd.), Naachtun - Uxul en el contexto de las tierras bajas mayas y más allá, Centro de estudios mayas, UNAM, México.

ARnAuld Marie-Charlotte

2008 "Maya urbanization, agrarian cities in a preindustrial world (Urbanización maya: ciudades agrarias en un mundo pre-industrial) », in A. G. Mastache, R. H. Cobean, A. García Cook, K. G. Hirth (éd.), Urbanism in MesoamericalEl urbanismo en Mesoamérica, INAH/Pennsylvania State University/ University Park, México, vol. II, pp. 1-36.

2011 «Fase y generación en la arquitectura residencial de Río Bec (Campeche) », communication présentée à « Los mayas y las concepciones del tiempo », L. Pescador (coord.), VIIa Mesa redonda de Palenque, 27 de noviembre2 de diciembre, INAH, México.

2014 «El proyecto maya de vida y sociedad», in F. de Pierrebourg, M. H. Ruz (coord.), Nah, otoch. Concepción, factura y atributos de la morada maya, 
Secretaria de Educación del Estado de Yucatán/UNAM/Fondo Mixto CONACYT-Gobierno del Estado de Yucatán, Izamal, pp. 37-64.

ARnauld Marie-Charlotte, Alfonso LaCADENa

2004 «Asentar su autoridad: banquetas en el Grupo B de Río Bec (Campeche, México) », Journal de la Société des américanistes, 90 (1), pp. 203-222.

Arnauld Marie-Charlotte, Dominique Michelet

2004 «Le développement des cités mayas », Annales, Histoire, Sciences sociales, $59^{\mathrm{e}}$ année, $\mathrm{n}^{\circ} 1$, pp. 78-103.

2010 «Casas monumentales de Río Bec, raíz del particularismo regional: variantes y variaciones ", in A. Monod-Becquelin, A. Breton, M. H. Ruz(éd.), Figuras mayas de la diversidad, CEPHCIS /UNAM, Mérida, pp. 409-431.

ARNAUld Marie-Charlotte, Philippe NondÉDÉo

2010 "Río Bec: between the Central and the Northern Lowlands », in L. van Broekhoven, R. Valencia R., B. Vis, F. Sachse (éd.), The Maya and their neighbours: internal and external contacts through time, Verlag Anton Saurwein (Acta Mesoamericana 22), Markt Schwaben, pp. 44-59.

Arnauld Marie-Charlotte, Sara Dzul G., Laure DÉodat

2010 "Evolución en la ocupación de los Grupos B y D de Río Bec (Campeche, México) », in A. Benavides C., E. Vargas P. (coord.), La península de Yucatán: investigaciones recientes y cronologías alternativas, Universidad autónoma de Campeche (Colección Arqueología, 7), Campeche, pp. 135-154.

ARnAuld Marie-Charlotte, Mélanie Forné, Sara Dzul

2007 «Dinámica entre unidades sociales vecinas: los Grupos A, B y D de Río Bec», communication présentée au symposium «Río Bec: génesis, desarrollo y percepción de un fenómeno cultural », P. Nondédéo (coord.), VIIo Congreso internacional de los mayistas, 8-14 de julio, Mérida.

Arnauld Marie-Charlotte, Dominique Michelet, Boris Vannière, Philippe NondéDÉo, Éva LEMONNIER

2012 «Houses, emulation and cooperation among the Rio Bec Groups", in M.-C. Arnauld, L. R. Manzanilla, M. E. Smith (éd.), The neighborhood as a social and spatial unit in Mesoamerican cities, University of Arizona Press, Tucson, pp. 202-228.

Arnauld Marie-Charlotte, Dominique Michelet, Philippe NondéDÉo

2013 "Living together in Río Bec Houses: co-residence, rank and alliance », Ancient Mesoamerica 24 (2), pp. 469-493.

BaLL Joseph W.

1977 The archaeological ceramics of Becan, Campeche, Mexico, Middle American Research Institute, Tulane University (Publication, 43), New Orleans.

2014 «Rethinking the Becán ceramic sequence: continuities, disjunctions, segmentation, and chronology », Latin American Antiquity, 25 (4), pp. 427-448. 
BAUDEZ Claude F.

2002 Une histoire de la religion des Mayas. Du panthéisme au panthéon, Albin Michel (Bibliothèque Histoire), Paris.

BECK Robin A. Jr.

2007 The durable house: house society models in archaeology, Center for Archaeological Investigations, Southern Illinois University (Occasional Paper, 35), Carbondale.

Becquelin Pierre, Dominique Michelet, Marie-Charlotte Arnauld, Grégory Pereira, Fabienne de Pierrebourg, Marie-France Fauvet-Berthelot, Philippe NONDÉDÉO, Éric TALADOIRE

2005 «La historia de Balamkú, Campeche, vista desde su Grupo Sur y su periferia ", in Los investigadores de la cultura maya 13, Tomo II, Universidad autónoma de Campeche/SEP/FOMES, Campeche, pp. 317-332.

Braswell Geoffrey E.

2013 «Ancient obsidian procurement and production in the Petén campechano: Uxul and Calakmul during the Early Classic to Terminal Classic periods », Indiana, 30, pp. 149-171.

Bueno Ricardo

1999 Entre un río de robles: un acercamiento a la arqueología de la región Río Bec, México, Instituto nacional de antropología e historia (Colección científica, 411), México.

Campaña Valenzuela Luz E., Edwin Ángulo Torres, Kenichiro Tsukamoto, Yareli JÁIDAR BENAVIDES

2004 Proyecto arqueológico Becán: informe anual de la temporada de campo 20032004, Archivo del Consejo de arqueología, INAH, México.

Carrasco Ramón, Sylviane Boucher, Agustín PeÑa

1986a «Río Bec: un modelo representativo del patrón de asentamiento regional », Boletín de la Escuela de ciencias antropológicas de la universidad de Yucatán, 13 (78), pp. 20-30.

1986b "Addenda a "Río Bec: un modelo representativo del patrón de asentamiento regional" ", Boletín de la Escuela de ciencias antropológicas de la universidad de Yucatán, 14 (79), p. 31.

Culbert T. Patrik, Don S. Rice (éd.)

1990 Precolumbian population history in the Maya Lowlands, University of New Mexico Press, Albuquerque.

DÉODAT Laure, Marie-Charlotte ARNAUld

2012 «Cocinas, comidas y convites en Río Bec», in P. Nondédéo, A. Breton (éd.), Maya daily lives, Verlag Anton Saurwein (Acta Mesoamericana, 24), Markt Schwaben, pp. 53-64.

\section{Drennan Robert D.}

1988 «Household location and compact versus dispersed settlement in Prehispanic Mesoamerica », in R. R. Wilk, W. Ashmore (éd.), Household and 
community in the Mesoamerican past, University of New Mexico Press, Albuquerque, pp. 273-293.

DunNING Nicholas P.

2004 "Down on the farm: Classic Maya "homesteads" as "farmsteads" ", in E. D. Lohse, F. Valdez Jr. (éd.), Ancient Maya commoners, University of Texas Press, Austin, pp. 97-116.

Dunning Nicholas P., Timothy P. Beach, Shery Luzzadder-Beach

2012 "Kax and kol: collapse and resilience in Lowland Maya civilization », Proceedings of the National Academy of Science, 109 (10), pp. 3652-3657.

Dussol Lydie

2012 L'Utilisation des bois de combustion dans les rites funéraires à Río Bec (Campeche, Mexique) au Classique Terminal (850-950 apr. J.-C.), mémoire de master 2, UFR Histoire de l'art et d'archéologie, université Paris 1 Panthéon-Sorbonne.

Dussol Lydie, Michelle Elliott, Grégory Pereira, Dominique Michelet

En préparation «The use of firewoods in ancient Maya funerary rituals: a case study from Río Bec (Campeche, Mexico)».

EARLE Thimothy

2000 "Archaeology, property, and prehistory », Annual Review of Anthropology, 29, pp. 39-60.

EATON Jack D.

1974 "Chicanna: an elite center in the Río Bec region », in Archaeological investigations on the Yucatan Peninsula, Middle American Research Institute, Tulane University (Publication, 31), New Orleans, pp. 133-138.

FARRISS Nancy M.

1984 Maya society under colonial rule. The collective enterprise of survival, Princeton University Press, Princeton.

Fowler William R., Marie-Charlotte Arnauld

2013 «Special section: noble farmers and weak kings in the Classic Maya Lowlands: the Río Bec archaeological project, 2002-2010. Introduction », Ancient Mesoamerica, 24 (2), pp. 343-351.

FREIDEL David

1981 "The political economics of residential dispersion among the Lowland Mayas ", in W. Ashmore (éd.), Lowland Maya settlement patterns, University of New Mexico Press (School of American Research advanced seminar series), Albuquerque, pp. 371-382.

Gillot Céline

2014 «The use of pozzolanic materials in Maya mortars: new evidence from Río Bec (Campeche, Mexico) », Journal of archaeological science, 47, pp. 1-9.

En préparation Architecture maya: étude des dimensions techniques et socioéconomiques de la production architecturale à Río Bec (Campeche, Mexique), 
entre 600 et 1000, thèse de doctorat, département d'Anthropologie, université de Montréal.

Gómez-Pompa Arturo, José S. Flores, Mario Aliphat F.

1990 «The sacred cacao groves of the Maya », Latin American Antiquity, 1 (3), pp. 247-257.

\section{HAMMOND Norman}

1978 "Cacao and cobaneros: an overland trade route between the Maya Highlands and Lowlands », in T. A. Lee, C. Navarrete (éd.), Mesoamerican communication routes and cultural contacts, New World Archaeological Foundation, Brigham Young University (Papers, 40), Provo, pp. 19-25.

HiRTH Kenneth G.

2008 "The economy of supply: modeling obsidian procurement and craft provisioning at a Central Mexican urban center ", Latin American Antiquity, 19 (4), pp. 435-457.

INOMATA Takeshi

2004 "The spatial mobility of non-elite populations in Classic Maya society and its political implications ", in E. D. Lohse, F. Valdez Jr. (éd.), Ancient Maya commoners, University of Texas Press, Austin, pp. 175-196.

JONES Grant D.

1982 " Agriculture and trade in the Colonial Period Southern Maya Lowlands ", in K. V. Flannery (éd.), Maya subsistence, Academic Press, New York, pp. 275-293.

Kennett Douglas J., Sebastian F. M. Breitenbach, Valorie V. Aquino, Yemane Asmerom, Jaime Awe, James U. L. Baldini, Patrick Bartlein, Brendan J. Culleton, Claire Ebert, Christopher Jazwa, Martha J. Macri, Norbert Marwan, Victor Polyak, Keith M. Prufer, Harriet E. Ridley, Harald Sodemann, Bruce Winterhalder, Gerald H. Haug

2012 «Development and disintegration of Maya political systems in response to climate change », Science, 338 (6108), 9 novembre, pp. 788-791.

KiLlion Thomas W. (éd.)

1992 Gardens of prehistory: the archaeology of settlement agriculture in Greater Mesoamerica, University of Alabama Press, Tuscaloosa.

LaCADENa Alfonso

2007 «Las inscripciones de Río Bec », communication présentée au symposium «Río Bec: génesis, desarrollo y percepción de un fenómeno cultural », P. Nondédéo (coord.), VIIo Congreso internacional de los mayistas, 8-14 de julio, Mérida.

LEMONNIER Éva

2009 La Structure de l'habitat du site maya classique de La Joyanca dans son environnement local (Petén, Guatemala), Archaeopress (BAR. International series, 2016 ; Paris monographs in American archaeology, 23), Oxford. 
LEMONNIER Éva, Boris VANNIÈRE

2007 «Medioambiente y territorios agrarios en la zona nuclear del sitio de Río Bec», communication présentée au symposium «Río Bec: génesis, desarrollo y percepción de un fenómeno cultural », P. Nondédéo (coord.), VIIo Congreso internacional de los mayistas, 8-14 de julio, Mérida.

2013 "Agrarian features, farmsteads, and homesteads in the Río Bec nuclear zone, Mexico », Ancient Mesoamerica, 24 (2), pp. 397-413.

LoHSE Jon C.

2004 «Intra-site settlement signatures and implications for Late Classic Maya commoner organization at Dos Hombres, Belize », in J. C. Lohse, F. Valdez Jr. (éd.), Ancient Maya commoners, University of Texas Press, Austin, pp. 117-145.

Marcus Joyce

2004 "Maya commoners: the stereotype and the reality », in J. C. Lohse, F. Valdez Jr. (éd.), Ancient Maya commoners, University of Texas Press, Austin, pp. 255-283.

\section{MarTin Simon}

2005 "Of snakes and bats: shifting identities at Calakmul », The PARI journal, $6(2)$, pp. 5-13.

McAnany Patricia A.

1995 Living with the ancestors: kinship and kingship in ancient Maya society, University of Texas Press, Austin.

1998 "Ancestors and the Classic Maya built environment », in S. D. Houston (éd.), Function and meaning in Classic Maya architecture, Dumbarton Oaks, Washington (D.C.), pp. 271-298.

McAnany Patricia A., Ben S. Thomas, Steven Morandi, Polly A. Peterson, Eleanor HARRISON

2002 "Praise the ajaw and pass the kakaw: Xibun Maya and the political economy of cacao », in M. A. Masson, D. A. Freidel (éd.), Ancient Maya political economies, Altamira Press, Walnut Creek/Lanham/New York/Oxford, pp. 123-139.

MERwin Raymond E.

1913 The ruins of the Southern part of the peninsula of Yucatan, with special reference to their place in the Maya area, thèse de doctorat, Harvard University, $125 \mathrm{p}$.

Michelet Dominique, Nicolas Latsanopoulos, Julie Patrois

2004a « ¿El ocaso de un estilo? Nota preliminar sobre la fachada norte del edificio con torres del Grupo A de Río Bec », Journal de la Société des américanistes, 90 (1), pp. 223-240.

Michelet Dominique, Éric Taladoire, Sara Dzul, Philippe Nondédéo, Julie Patrois, Marie-Charlotte Arnauld, Angeles Cantero, Pierre Becquelin, Rosario Acosta, Emyly González, Diana Arano, Alfonso Lacadena, Didier Galop, Boris VanNiÈre 
2004b Proyecto Río Bec (Campeche, Mexique). Informe de la segunda temporada, del 27 de febrero al 25 de mayo de 2003, CNRS/CEMCA, Paris/México.

Michelet Dominique, Éric Taladoire, Sara Dzul, Philippe Nondédéo, Julie Patrois, Georges Marchand, Marie-Charlotte Arnauld, Laure Déodat, Astrid Huser, Pierre Becquelin, Grégory Pereira, Jorge Ortega, Emyly González, Ma. Rosalía Carrillo, Diana Arano, Didier Galop, Boris VANniÈre, Chloé ANDrieu

2005 Proyecto Río Bec (Campeche México). Informe de la tercera temporada, del 16 de febrero al 15 de mayo de 2004, CNRS/CEMCA, Paris/México.

Michelet Dominique, Éric Taladoire, Sara Dzul, Philippe Nondédéo, Julie Patrois, Marisa Vásquez, Alfonso Lacadena, Georges Marchand, MarieCharlotte Arnauld, Laure Déodat, Sophie Lardé, Céline Gillot, Grégory Pereira, Amparo Robles, Emyly González, Ma. Rosalía Carrillo, Diana Arano, Didier Galop, Boris Vannière, Christine Heinz, Nicolas LATSANOPOULOS

2006 Proyecto Río Bec (Campeche México). Informe de la cuarta temporada, del 8 de febrero al 6 de mayo de 2005, CNRS/CEMCA, Paris/México.

Michelet Dominique, Marie-Charlotte Arnauld, Philippe Nondédéo, Éric Taladoire, Sara Dzul, Chloé Andrieu, Laure Déodat, Céline Gillot, Julie Patrois, Grégory Pereira, J. Joel Hernández O., Georges Marchand, Agnès Stock, Boris Vannière, Éva Lemonnier, Christine Heinz, Emyly GonzÁlez, Ma. Rosalia CARrillo, Yareli Jaídar, Nicolas Latsanopoulos

2007 Proyecto Río Bec (Campeche México). Informe de la quinta temporada, del 5 de febrero al 4 de mayo de 2006, CNRS/CEMCA, Paris/México.

Michelet Dominique, Marie-Charlotte Arnauld, Philippe Nondédéo, Éric Taladoire, Sara Dzul, Chloé Andrieu, Laure Déodat, Céline Gillot, Julie Patrois, Alfonso Lacadena, Ignacio Cases, Grégory Pereira, José D. Álvarez, Gabriel Francia G., Éva Lemonnier, Emyly González, Ma. Rosalía CARrillo, Yareli Jaídar, Adela VÁsquez, Nicolas Latsanopoulos

2008 Proyecto Río Bec (Campeche México). Informe de la sexta temporada, del 4 de febrero al 3 de mayo de 2007, CNRS/CEMCA, Paris/México.

Michelet Dominique, Philippe Nondédéo, Julie Patrois, Céline Gillot, Emyly González 2013 «Structure 5N2 (Group A): a Río Bec paradigmatic palace? », Ancient Mesoamerica, 24 (2), pp. 415-431.

Nalda Enrique, Luz E. CAmpaña V.

1998 «Dzibanché: una alternativa de interpretación del patrón de asentamiento del sur de Quintana Roo », in S. Trejo (éd.), Modelos de entidades politicas mayas. Primer seminario de mesas redondas de Palenque, Conaculta/INAH, México, pp. 5-56.

NondÉDÉo Philippe, Sara Dzul

2010 "Arquitectura y cronología: hacia un esquema preliminar de evolución en la ocupación de la microrregión de Río Bec », in A. Benavides C., E. Vargas 
(coord.), Lapenínsula de Yucatán: investigaciones recientes y cronologías alternativas, Universidad autónoma de Campeche (Colección Arqueología, 7), Campeche, pp. 91-120.

NondÉDÉo Philippe, Alfonso LaCADENA

2004 "Kajtún: un nuevo sitio maya con monumentos esculpidos en la región Río Bec », Journal de la Société des américanistes, 90 (1), pp. 183-201.

NondéDÉo Philippe, Julie PatroIS

2007 «Iconografía del poder en la región Río Bec: representaciones y primeras interpretaciones », in E. Vargas, A. Benavides (éd.), El patrimonio arqueológico maya en Campeche. Novedades, afectaciones, soluciones, UNAM (Cuadernos del Centro de estudios mayas, 35), México, pp. 159-206.

2010 "Variaciones en la morfología y la decoración de los edificios mayores de Río Bec: en busca de un significado », in A. Monod-Becquelin, A. Breton, M. H. Ruz (éd.), Figuras mayas de la diversidad, CEPHCIS/UNAM, Mérida, pp. 305-354.

NondédÉo Philippe, Dominique Michelet, Marie-Charlotte Arnauld, Éric TaladoIre, Julie Patrois, Ramzy Barrois

2002 Proyecto Río Bec (Campeche México). Informe de la primera temporada, del 15 de febrero al 18 de mayo de 2002, CNRS/CEMCA, Paris/México.

NondédÉo Philippe, Julie Patrois, Alfonso LaCAdena, Marie-Charlotte Arnauld, Éric TAladoire, Dominique Michelet

2010 « De la autonomía política y cultural de la provincial de Río Bec », Estudios de cultura maya, XXXVI, pp. 37-66.

NondéDÉo Philippe, Marie-Charlotte Arnauld, Dominique Michelet

2013 «Río Bec settlement patterns and local socio-political organization », Ancient Mesoamerica, 24 (2), pp. 373-396.

OKoshi Tsubasa

2006 «Kax (monte) y luum (tierra): la transformación de los espacios mayas en el siglo XVI », in K. Ochiai (coord.), El mundo maya: miradas japonesas, UNAM, México, pp. 85-105.

2012 "Post-Classic Maya "barrios" in Yucatan: a historical approach », in M.-C. Arnauld, L. R. Manzanilla, M. E. Smith (éd.), The neighborhood as a social and spatial unit in Mesoamerican cities, University of Arizona Press, Tucson, pp. 331-351.

Palerm Angel, Eric Wolf

1972 Agricultura y civilización en Mesoamérica, Secretaría de Educación Pública (SepSetentas, 32), México.

Patrois Julie

2013 «Río Bec graffiti: a private form of art, Ancient Mesoamerica, 24 (2), pp. 433-447.

PEREIRA Grégory

2007 "Costumbres funerarias en Río Bec: primeros resultados », communication présentée au symposium « Río Bec: génesis, desarrollo y percepción de un 
fenómeno cultural », P. Nondédéo (coord.), VIIo Congreso internacional de los mayistas, 8-14 de julio, Mérida.

2013 «Ash, dirt and rock: burial practices at Río Bec », Ancient Mesoamerica, 24 (2), pp. 449-468.

PÉRIGNY Maurice de

1908 "Yucatan inconnu », Journal de la Société des américanistes, 5, pp. 67-84.

PoPE Jeffrey, DAHLIN Bruce

1989 «Ancient Maya wetland agriculture: new insights from ecological and remote sensing research », Journal of field archaeology, 16 (1), pp. 87-106.

ROVNER Irwin, Suzanne LEwENSTEIN

1997 Maya stone tools of Dzibilchaltun, Yucatán, and Becan and Chicanna, Campeche, Middle American Research Institute, Tulane University (Publication, 65), New Orleans.

Roys Ralph L.

1943 The Indian background of Colonial Yucatan, Carnegie Institution (Carnegie Institution of Washington Publication, 548), Washington (D.C.).

Ruppert Karl, John H. Denison Jr.

1943 Archaeological reconnaissance in Campeche, Quintana Roo, and Peten, Carnegie Institution (Carnegie Institution of Washington Publication, 543), Washington (D.C.).

SAPPER Karl

1895a « Die unabhängigen Indianerstaaten von Yucatán », Globus, 67, pp. 197-201.

1895b «The old Indian settlements and architectural structures in Northern Central America », Annual report of the Smithsonian Institution, pp. 537-555.

SEUFFERT Andy

1974 «El templo B redescubierto en la zona de Río Bec », Boletín del INAH, época 2, 8, pp. 3-18.

SheETs Payson

1992 The Ceren site. A prehistoric village buried by volcanic ash in Central America, Harcourt Brace Jovanovich College Publishers (Case studies in archaeology series), Fort Worth.

Sion Julien

2010 La Structure 5N2bis, Río Bec (Campeche, Mexique) : caractérisation fonctionnelle et étude de son mobilier céramique. Une cuisine chez des Mayas des Basses Terres au Classique Récent/Terminal, mémoire de master 2, UFR d'histoire de l'art et d'archéologie, université de Paris 1 Panthéon-Sorbonne.

Stark Barbara, Alanna Ossaz

2007 «Ancient settlement, urban gardening, and environment in the Gulf Lowlands of Mexico », Latin American Antiquity, 18 (4), pp. 385-406. 
TALADOIRE Éric

2007a Río Bec, síntesis de la Operación II: propuesta de fechamiento de los sondeos, manuscrit, archives du projet Río Bec, Nanterre.

2007b Posibles implicaciones de la ocupación espacial en Río Bec a partir de la cronología, manuscrit, archives du projet Río Bec, Nanterre.

TAladoire Éric, Sara Dzul, Philippe Nondédéo, Mélanie Forné

2013 "Chronology of the Río Bec settlement and architecture", Ancient Mesoamerica, 24 (2), pp. 353-372.

Thomas Prentice M. Jr.

1981 Prehistoric Maya settlement patterns at Becan, Campeche, Mexico, Middle American Research Institute, Tulane University (Publication, 45), New Orleans.

TURNER II Bill L.

1983 Once beneath the forest: prehistoric terracing in the Rio Bec region of the Maya Lowlands, Westview Press, Boulder (Col.).

TURner II Bill L., Jeremy A. SABLOFF

2012 "Classic Period collapse of the Central Maya Lowlands: insights about human-environment relationships for sustainability ", Proceedings of the National Academy of Science, 109 (35), pp. 13908-13914.

VAPNARSKY Valentina, Olivier LE GuEN

2011 «The guardians of space and history: understanding ecological and historical relations of the contemporary Yucatec Maya to their landscape ", in C. Isendahl, B. Liljefors Persson (éd.), Ecology, power, and religion in Maya landscapes, Verlag Anton Saurwein (Acta Mesoamericana, 23), Markt Schwaben, pp. 191-206.

WeBster David L.

1976 Defensive eathworks at Becán, Campeche, Mexico. Implications for Maya warfare, Middle American Research Institute, Tulane University (Publication, 41), New Orleans.

2005 «Political ecology, political economy, and the culture history of resource management at Copan », in A. E. Wyllys, W. L. Fash, (éd.), Copán. The history of an ancient Maya kingdom, School of American research/James Currey, Santa Fe/Oxford, pp. 33-72.

WeLls Christian E.

2006 "Recent trends in theorizing prehispanic Mesoamerican economies », Journal of archaeological research, 14, pp. 265-312.

WILK Richard R.

1988 "Maya household organization: evidence and analogies », in R. R. Wilk, W. Ashmore (éd.), Household and community in the Mesoamerican Past, University of New Mexico Press, Albuquerque, pp. 135-151. 